\title{
ON THE DECOMPOSITION MATRICES OF THE QUANTIZED SCHUR ALGEBRA
}

\author{
Michela VARAGNOLO and Eric VASSEROT ${ }^{1}$
}

\begin{abstract}
We prove the decomposition conjecture for the Schur algebra stated in $[\mathrm{LT}]$. We also give a new approach to the Lusztig conjecture via canonical bases of the Hall algebra.
\end{abstract}

\section{Introduction and general notations.}

0.1. The aim of this paper is to give a proof of the decomposition conjecture for the quantized Schur algebra [LT, Conjecture 5.2] which generalizes the theorem of Ariki (see $[\mathrm{A}]$ ) on the decomposition numbers of the Hecke algebra of type $A$. More precisely, let $\bigwedge^{\infty}$ be the level 1 Fock space of type $A$ and let $\mathbf{B}^{ \pm}$be the bases of $\bigwedge^{\infty}$ introduced in [LT]. The decomposition conjecture links the decomposition matrices of the quantized Schur algebra and the basis $\mathbf{B}^{+}$. Our proof consists in two steps : first we express $\mathbf{B}^{ \pm}$in terms of some Kazhdan-Lusztig polynomials. Then we note that a simple module of the quantized Schur algebra can be pulled-back to a simple module of the Lusztig integral form of the quantized enveloping algebra of $\mathfrak{s l}_{k}$ (denoted by $\mathbf{U}\left(\mathfrak{s l}_{k}\right)$ ). Thus, the Lusztig conjecture for the dimension of the simple $\mathbf{U}\left(\mathfrak{s l}_{k}\right)$-modules at roots of unity identifies the entries of the decomposition matrices with some Kazhdan-Lusztig polynomials. It suffices to observe that these polynomials are precisely the ones which appear in $\mathbf{B}^{+}$.

Let $\mathbf{U}_{n}^{-}$be the Hall algebra of nilpotent representations of the cyclic quiver. Set $\varepsilon=\exp \left(2 i \pi / n^{\prime}\right)$. Put $n=n^{\prime}$ if $n$ odd, $n=n^{\prime} / 2$ else, i.e. $n$ is the order of $\varepsilon^{2}$. Let $\mathbf{U}_{\varepsilon}\left(\mathfrak{s l}_{k}\right)$ be the specialization at $v=\varepsilon$ of $\mathbf{U}\left(\mathfrak{s l}_{k}\right)$. We give a new approach to the proof of the Lusztig conjecture on the character of the simple modules of $\mathbf{U}_{\varepsilon}\left(\mathfrak{s l}_{k}\right)$ in terms of the canonical basis of $\mathbf{U}_{n}^{-}$. Recall that this conjecture (proved by Kashiwara-Tanisaki and Kazhdan-Lusztig) gives the multiplicity of the Weyl module of $\mathbf{U}_{\varepsilon}\left(\mathfrak{s l}_{k}\right)$ with highest weight $\mu$, say $W_{\mu}$, in the simple $\mathbf{U}_{\varepsilon}\left(\mathfrak{s l}_{k}\right)$-module with highest weight $\lambda$, say $V_{\lambda}$, i.e.

$$
\left[V_{\lambda}: W_{\mu}\right]=\sum_{y}(-1)^{l(y x)} P_{y x}(1)
$$

where $x \in \widehat{\mathfrak{S}}_{k}$ is minimal such that $\nu=\lambda \cdot x^{-1}$ satisfies

$$
\nu_{i}<\nu_{i+1} \quad \forall i=1,2, \ldots, k-1, \quad \nu_{1}-\nu_{k} \geq 1-k-n,
$$

\footnotetext{
${ }^{1}$ Both authors are partially supported by the EEC grant no. ERB FMRX-CT97-0100.
} 
and $\mu=\lambda \cdot x^{-1} y$. We proceed as follows. First we prove that $\bigwedge^{\infty}$ is a cyclic $\mathbf{U}_{n}^{-}$module generated by the vacuum vector $|\emptyset\rangle$. Then we define a basis $\mathbf{B}^{\prime}$ of $\mathbf{U}_{n}^{-}$using intersection cohomology. We construct a basis $\mathbf{B}$ of $\bigwedge^{\infty}$ via the action of $\mathbf{B}^{\prime}$ on the vacuum vector. We prove that $\mathbf{B}$ and $\mathbf{B}^{+}$are fixed by the same semi-linear involution (see Theorem 6.3). At last, we prove that the equality $\mathbf{B}=\mathbf{B}^{+}$is a $q$-analogue of the Lusztig conjecture (see Subsection 11.4). The reader should be warned that we endow the Hall algebra with the product opposit to the usual one (used in [G1] or [L1-4]).

The plan of the paper is the following. In Sections 1-4 we recall the definitions and the main properties of the basic objects. In Sections 5-6 we construct an action of $\mathbf{U}_{n}^{-}$on the Fock space $\bigwedge^{\infty}$. Proposition 6.1 is new. In Section 7 we introduce the convolution algebra on pairs of affine flags. This algebra is a geometric analogue of the affine Schur algebra (Proposition 7.4) and is related to $\mathbf{U}_{n}^{-}$in Proposition 7.6. In Sections 8-9 we give a representation of $\mathbf{U}_{n}^{-}$on the finite wedges space, $\bigwedge^{l}$, via the coproduct of $\mathbf{U}_{n}^{-}$. This action is related to the convolution algebra on affine flags by Lemma 8.3. In Section 10 we interpret the action of $\mathbf{U}_{n}^{-}$on $\bigwedge^{\infty}$ as a "limit" of $\bigwedge^{l}$ when $l$ goes to infinity. Using the results of Sections 7-9 we prove that the elements of $\mathbf{B}$ are fixed by the Leclerc-Thibon involution (Theorem 6.3). In Section 11 we prove the Decomposition Conjecture. Let us observe that the proof only uses the results of Sections 8 and 9. In Section 12 we reinterpret the Lusztig conjecture. We use in an essentiel way the construction of the representation of $\mathbf{U}_{n}^{-}$on $\bigwedge^{\infty}$ given in Section 6 .

\section{Contents.}
1. The Hecke algebra.
2. The quantum group.
3. The Hall algebra.
4. The Fock space.
5. The representation of $\mathrm{U}_{\infty}^{-}$on $\bigwedge^{\infty}$.
6. The representation of $\mathrm{U}_{n}^{-}$on $\bigwedge^{\infty}$.
7. Flag varieties.
8. The tensor representation of $\tilde{\mathbf{U}}_{n}^{-}$.
9. The action of $\mathbf{U}_{n}^{-}$on wedges.

10. Proof of Theorem 6.3.

11. Proof of the Decomposition Conjecture.

12. The Lusztig conjecture.

13. Proof of Proposition 6.1.

0.2. We now fix a few general notations. Set $\mathbb{S}=\mathbb{C}[v], \mathbb{A}=\mathbb{C}\left[v, v^{-1}\right]$. Let $\mathbb{F}$ be a field with $q^{2}$ elements and let $\overline{\mathbb{F}}$ be the algebraic closure of $\mathbb{F}$. Fix a set $I$. For any $i \in I$ and $r \in \mathbb{N}^{\times}$, let $\overline{\mathbb{F}}^{r}[i]$ be the $I$-graded $\overline{\mathbb{F}}$-vector space with a single $r$-dimensional component, in degree $i$. Let $\epsilon_{i} \in \mathbb{N}^{(I)}$ be the dimension of $\overline{\mathbb{F}}[i]$. For any $d \in \mathbb{N}^{(I)}$ set $|d|=\sum_{i \in I} d_{i}$. If $i \in \mathbb{Z}$ let $\bar{\imath}$ be the class of $i$ in $\mathbb{Z} / n \mathbb{Z}$. Given a positive integer $l$ let $\Pi(l)$ be the set of all the partitions of $l$ and let $\Pi_{l}$ be the set of partitions with at most $l$ parts. Put $\Pi=\cup_{l} \Pi(l)$. The set $\Pi$ is endowed with the usual order. If $\lambda \in \Pi$ let $\lambda^{\prime}$ be the dual partition. For an irreducible algebraic variety $X$ we denote by $\mathcal{H}^{i}\left(I C_{X}\right)$ the $i$-th cohomology sheaf of the intersection complex of $X$. Then, for any stratum $Y \subset X$, let $\operatorname{dim} \mathcal{H}_{Y}^{i}\left(I C_{X}\right)$ be the dimension 
of the stalk of $\mathcal{H}^{i}\left(I C_{X}\right)$ at a point of $Y$. For any set $X$ with the action of a group $G$ let $\mathbb{C}_{G}(X)$ be the set of $G$-invariant functions $X \rightarrow \mathbb{C}$ supported on a finite number of orbits. For any subset $X$ of an algebraic variety let $\bar{X}$ denote its Zariski closure.

\section{The Hecke algebra.}

1.1. Fix $n \in \mathbb{N}^{\times}$and set

$$
A_{l}^{n}=\left\{\mathbf{i} \in \mathbb{Z}^{l} \mid 1-n \leq i_{1} \leq i_{2} \leq \cdots \leq i_{l} \leq 0\right\}
$$

Let $\mathfrak{S}_{l}$ be the symmetric group and let $\widehat{\mathfrak{S}}_{l}=\mathfrak{S}_{l} \ltimes \mathbb{Z}^{l}$ be the extended affine Weyl group. Let $\widehat{S}_{l} \subset \widehat{\mathfrak{S}}_{l}$ be the set of simple affine reflexions and put $S_{l}=\widehat{S}_{l} \cap \mathfrak{S}_{l}$. As usual, the simple affine reflexions are denoted by $s_{0}, s_{1}, \ldots, s_{l-1}$ in such a way that $S_{l}=\left\{s_{1}, s_{2}, \ldots, s_{l-1}\right\}$. Let $\pi \in \widehat{\mathfrak{S}}_{l}$ be the zero length element such that $s_{i-1}=\pi^{-1} s_{i} \pi$. The group $\widehat{\mathfrak{S}}_{l}$ acts on $\mathbb{Z}^{l}$ on the right in such a way that

$$
\begin{array}{ll}
\text { (i) } \lambda=\mathbf{i}+n \lambda & \text { if } \quad \lambda \in \mathbb{Z}^{l} \\
\text { (i) } s_{j}=\left(i_{1}, i_{2}, \ldots, i_{j+1}, i_{j}, \ldots, i_{l}\right) & \text { if } \quad j \neq 0 \\
\text { (i) } s_{0}=\left(i_{l}-n, i_{2}, \ldots, i_{l-1}, i_{1}+n\right) . &
\end{array}
$$

The alcove $A_{l}^{n}$ is a fundamental domain for this action. If $\mathbf{i} \in A_{l}^{n}$ let $\mathfrak{S}_{\mathbf{i}} \subset \widehat{\mathfrak{S}}_{l}$ be its isotropy group, $S_{\mathbf{i}}=\widehat{S}_{l} \cap \mathfrak{S}_{\mathbf{i}}$, and let $\mathfrak{S}^{\mathbf{i}}$ be the set of minimal length representatives of the cosets in $\mathfrak{S}_{\mathbf{i}} \backslash \widehat{\mathfrak{S}}_{l}$. For any $x \in \widehat{\mathfrak{S}}_{l}$, let $x_{\mathbf{i}} \in \mathfrak{S}_{\mathbf{i}}$ and $x^{\mathbf{i}} \in \mathfrak{S}^{\mathbf{i}}$ be such that $x=x_{\mathbf{i}} x^{\mathbf{i}}$. Let $\omega \in \mathfrak{S}_{l}$ be the longest element. Set $\rho=(0,-1,-2, \ldots, 1-l) \in \mathbb{Z}^{l}$ and put

$$
\lambda \cdot x=(\lambda+\rho) x-\rho, \quad x \in \widehat{\mathfrak{S}}_{l}, \quad \forall \lambda \in \mathbb{Z}^{l} .
$$

1.2. The Hecke algebra of type $G L_{l}$, say $\mathbf{H}_{l}$, is the unital associative $\mathbb{A}$-algebra generated by $T_{i}^{ \pm 1}, i=1,2, \ldots l-1$ modulo the following relations

$$
T_{i} T_{i}^{-1}=1=T_{i}^{-1} T_{i}, \quad\left(T_{i}+1\right)\left(T_{i}-v^{-2}\right)=0,
$$

$$
T_{i} T_{i+1} T_{i}=T_{i+1} T_{i} T_{i+1}, \quad|i-j|>1 \Rightarrow T_{i} T_{j}=T_{j} T_{i} .
$$

The affine Hecke algebra of type $G L_{l}$, say $\widehat{\mathbf{H}}_{l}$, is the unital associative $\mathbb{A}$-algebra generated by $T_{i}^{ \pm 1}, X_{j}^{ \pm 1}, i=1,2, \ldots, l-1, j=1,2, \ldots, l$ modulo the relations $(a)$ and

$$
\begin{gathered}
X_{i}, X_{i}^{-1}=1=X_{i}^{-1} X_{i}, \quad X_{i} X_{j}=X_{j} X_{i}, \\
T_{i} X_{i} T_{i}=v^{-2} X_{i+1}, \quad j \neq i, i+1 \Rightarrow X_{j} T_{i}=T_{i} X_{j} .
\end{gathered}
$$

For all $x \in \mathfrak{S}_{l} \ltimes \mathbb{Z}^{l}$ let $l(x)$ be the length of $x$ and let $\tilde{T}_{x}$ be the normalized element $\tilde{T}_{x}=v^{l(x)} T_{x}$. The algebra $\widehat{\mathbf{H}}_{l}$ is isomorphic to the Hecke algebra of the extended affine Weyl group $\mathfrak{S}_{l} \ltimes \mathbb{Z}^{l}$ via the Bernstein isomorphism which maps $\tilde{T}_{\lambda}^{-1}$ to $X^{\lambda}=X_{1}^{\lambda_{1}} X_{2}^{\lambda_{2}} \cdots X_{l}^{\lambda_{l}}$ if $\lambda \in \mathbb{Z}^{l}$ is dominant, i.e. if $\lambda_{1} \geq \lambda_{2} \geq \cdots \geq \lambda_{l}$. The semilinear involution ${ }^{-}: \widehat{\mathbf{H}}_{l} \rightarrow \widehat{\mathbf{H}}_{l}$ is such that $\bar{T}_{x}=T_{x^{-1}}^{-1}$ for all $x$. For all $x$ put $\tilde{T}_{x}=v^{l(x)} T_{x}$. If $t \in \mathbb{C}^{\times}$let $\widehat{\mathbf{H}}_{l \mid t}$ be the specialization of $\widehat{\mathbf{H}}_{l}$ at $v=t$. 


\section{The quantum group.}

Put $I=\{1,2, \ldots, n-1\}$ (resp. $I=\{0,1, \ldots, n-1\}$ ) and let $a_{i j}$ be the entries of the Cartan matrix of type $A_{n-1}$ (resp. $A_{n-1}^{(1)}$ ). The quantized enveloping enveloping algebra of $\mathfrak{s l}_{n}\left(\right.$ resp. $\left.\widehat{\mathfrak{s l}}_{n}\right)$ is the unital associative $\mathbb{C}(v)$-algebra generated by $\mathbf{e}_{i}, \mathbf{f}_{i}, \mathbf{k}_{i}^{ \pm 1}, i \in I$, modulo the Kac-Moody type relations

$$
\begin{gathered}
\mathbf{k}_{i} \mathbf{k}_{i}^{-1}=1=\mathbf{k}_{i}^{-1} \mathbf{k}_{i}, \quad \mathbf{k}_{i} \mathbf{k}_{j}=\mathbf{k}_{j} \mathbf{k}_{i}, \\
\mathbf{k}_{i} \mathbf{e}_{j}=v^{a_{i j}} \mathbf{e}_{j} \mathbf{k}_{i}, \quad \mathbf{k}_{i} \mathbf{f}_{j}=v^{-a_{i j}} \mathbf{f}_{j} \mathbf{k}_{i}, \quad\left[\mathbf{e}_{i}, \mathbf{f}_{j}\right]=\delta_{i j} \frac{\mathbf{k}_{i}-\mathbf{k}_{i}^{-1}}{v-v^{-1}}, \\
\sum_{k=0}^{1-a_{i j}}(-1)^{k} \mathbf{e}_{i}^{(k)} \mathbf{e}_{j} \mathbf{e}_{i}^{\left(1-a_{i j}-k\right)}=\sum_{k=0}^{1-a_{i j}}(-1)^{k} \mathbf{f}_{i}^{(k)} \mathbf{f}_{j} \mathbf{f}_{i}^{\left(1-a_{i j}-k\right)}=0 \quad \text { if } \quad i \neq j,
\end{gathered}
$$

where

$$
[k]=\frac{v^{k}-v^{-k}}{v-v^{-1}}, \quad[k] !=[k][k-1] \cdots[1], \quad \mathbf{e}_{i}^{(k)}=\frac{\mathbf{e}_{i}^{k}}{[k] !}, \quad \mathbf{f}_{i}^{(k)}=\frac{\mathbf{f}_{i}^{k}}{[k] !} .
$$

We denote by $\mathbf{U}\left(\mathfrak{s l}_{n}\right)$ (resp. $\mathbf{U}\left(\widehat{\mathfrak{s l}}_{n}\right)$ ) the Lusztig integral form, i.e. the $\mathbb{A}$-subalgebra generated by the divided powers $\mathbf{e}_{i}^{(k)}, \mathbf{f}_{i}^{(k)}$, and by $\mathbf{k}_{i}^{ \pm 1}$. If $n=\infty$ the algebra $\mathbf{U}\left(\mathfrak{s l}_{\infty}\right)$ is well defined. The algebras above are Hopf algebras. The coproduct is

$$
\Delta \mathbf{e}_{i}=\mathbf{e}_{i} \otimes \mathbf{k}_{i}+1 \otimes \mathbf{e}_{i}, \quad \Delta \mathbf{f}_{i}=\mathbf{f}_{i} \otimes 1+\mathbf{k}_{i}^{-1} \otimes \mathbf{f}_{i}, \quad \Delta \mathbf{k}_{i}=\mathbf{k}_{i} \otimes \mathbf{k}_{i} .
$$

Let $\mathbf{U}^{-}\left(\widehat{\mathfrak{s l}}_{n}\right) \subset \mathbf{U}\left(\widehat{\mathfrak{s l}}_{n}\right)$ and $\mathbf{U}^{-}\left(\mathfrak{s l}_{\infty}\right) \subset \mathbf{U}\left(\mathfrak{s l}_{\infty}\right)$ be the subalgebras generated by the elements $\mathbf{f}_{i}^{(k)}$.

\section{The Hall algebra.}

In this section we recall some of the results of [L1-4] and [G1].

3.1. Fix a finite field $\mathbb{F}$ with $q^{2}$ elements as in the introduction. Let $\Gamma=(I, J)$ be an oriented graph : $I$ is the set of vertices and $J$ is the set of arrows. Given an arrow $j \in J$ let $j_{1}$ and $j_{2}$ be respectively the input vertex and the output vertex. Fix $d \in \mathbb{N}^{(I)}$ and let $V$ be an $I$-graded $\mathbb{F}$-vector space of dimension $d$. Let $E_{V} \subseteq \bigoplus_{j \in J} \operatorname{Hom}\left(V_{j_{1}}, V_{j_{2}}\right)$ be the subset of nilpotent representations of $\Gamma$ on $V$. In this paper we will suppose that $\Gamma$ is one of the following two graphs:

(a) $\Gamma=\Gamma_{n}$ is the cyclic quiver of type $A_{n}^{(1)}$, i.e. $I=\mathbb{Z} / n \mathbb{Z}$ and $J=\{\bar{\imath} \rightarrow \bar{\imath}+1 \mid \bar{\imath} \in$ $\mathbb{Z} / n \mathbb{Z}\}$

(b) $\Gamma=\Gamma_{\infty}$ is the infinite quiver of type $A_{\infty}$, i.e. $I=\mathbb{Z}$ and $J=\{i \rightarrow i+1 \mid i \in \mathbb{Z}\}$.

3.2. Set $\mathbf{A}_{d}=\mathbb{C}_{G_{V}}\left(E_{V}\right)$ where $G_{V}=\prod_{i \in I} G L\left(V_{i}\right)$. Given $a, b \in \mathbb{N}^{(I)}$ such that $d=a+b$, fix $I$-graded $\mathbb{F}$-vector spaces $U, W$ of dimensions $a, b$. Let consider the diagram

$$
E_{U} \times E_{W} \stackrel{p_{1}}{\stackrel{p_{2}}{\longrightarrow}} F \stackrel{p_{3}}{\longrightarrow} E_{V},
$$

where 
(c) $E$ is the set of triples $(x, \phi, \psi)$ such that $x \in E_{V}$,

$$
0 \rightarrow U \stackrel{\phi}{\longrightarrow} V \stackrel{\psi}{\longrightarrow} W \rightarrow 0
$$

is an exact sequence of $I$-graded vector spaces and $\phi(U)$ is stable by $x$,

(d) $F$ is the set of pairs $\left(x, U^{\prime}\right)$ where $x \in E_{V}$ and $U^{\prime} \subset V$ is a $x$-stable $I$-graded subspace of dimension $a$.

Given $f \in \mathbb{C}_{G_{U}}\left(E_{U}\right)$ and $g \in \mathbb{C}_{G_{W}}\left(E_{W}\right)$ set

$$
f \circ g=q^{-m(b, a)}\left(p_{3}\right) ! h \in \mathbb{C}_{G_{V}}\left(E_{V}\right)
$$

where $h \in \mathbb{C}(F)$ is the function such that $p_{2}^{*} h=p_{1}^{*}(f g)$ and $m(b, a)=\sum_{j \in J} b_{j_{1}} a_{j_{2}}+$ $\sum_{i \in I} b_{i} a_{i}$. Then $(\mathbf{A}, \circ)$, where $\mathbf{A}=\bigoplus_{d} \mathbf{A}_{d}$, is an associative algebra.

3.3. Given $a, b \in \mathbb{N}^{(J)}$ such that $d=a+b$, fix a $I$-graded $\mathbb{F}$-vector space $U \subset V$ of dimension $a$. Let consider the diagram

$$
E_{U} \times E_{V / U} \stackrel{p}{\stackrel{p}{ }} E \stackrel{i}{\longrightarrow} E_{V} .
$$

Here $E \subset E_{V}$ is the subset of representations preserving $U$, the map $i$ is the inclusion and $p$ is the obvious projection. Set

$$
\Delta_{a, b}: \mathbf{A}_{d} \rightarrow \mathbf{A}_{a} \otimes \mathbf{A}_{b}, \quad f \mapsto q^{-n(b, a)} p_{!} i^{*} f,
$$

where $n(b, a)=\sum_{j \in J} b_{j_{1}} a_{j_{2}}-\sum_{i \in I} b_{i} a_{i}$.

3.4. Recall that $\Gamma=\Gamma_{n}$ or $\Gamma_{\infty}$. The classification of the isomorphism classes of nilpotent representations of $\Gamma$ does not depend on the ground field $\mathbb{F}$. It is proved in $[R]$ that the structural constants of $\mathbf{A}$ in the basis formed by the characteristic functions of the $G_{V}$-orbits in $E_{V}$ are the value at $v=q$ of universal polynomials in $\mathbb{A}$. Thus $\mathbf{A}$ can be viewed as the specialization at $v=q$ of a $\mathbb{A}$-algebra, called the generic Hall algebra. Let $\mathbf{U}_{n}^{-}$(resp. $\mathbf{U}_{\infty}^{-}$) be the generic Hall algebra if $\Gamma=\Gamma_{n}$ (resp. $\left.\Gamma=\Gamma_{\infty}\right)$. It is known that $\mathbf{U}_{\infty}^{-}$is isomorphic to $\mathbf{U}^{-}\left(\mathfrak{s l}_{\infty}\right)$ and that $\mathbf{U}^{-}\left(\widehat{\mathfrak{s l}}_{n}\right)$ embeds in $\mathbf{U}_{n}^{-}$(see [G1]). Let $\mathbf{A}^{0}$ be the $\mathbb{A}$-linear span of elements $\mathbf{k}_{d}$ with $d \in \mathbb{Z}^{(I)}$ such that

$$
\mathbf{k}_{0}=1 \quad \text { and } \quad \mathbf{k}_{a} \mathbf{k}_{b}=\mathbf{k}_{a+b}, \quad \forall a, b .
$$

For simplicity we will write $\mathbf{k}_{i}=\mathbf{k}_{\epsilon_{i}}$ for all $i \in I$. Set $\tilde{\mathbf{A}}=\mathbf{A} \otimes_{\mathbb{A}} \mathbf{A}^{0}$ and put

$$
\left(f \otimes \mathbf{k}_{a}\right) \circ\left(g \otimes \mathbf{k}_{b}\right)=v^{-a \cdot d}(f \circ g) \otimes \mathbf{k}_{a+b}, \quad \forall g \in \mathbf{A}_{d} \quad \forall f \in \mathbf{A},
$$

where $a \cdot d=-n(a, d)-n(d, a)$. Consider the map $\Delta: \tilde{\mathbf{A}} \rightarrow \tilde{\mathbf{A}} \otimes_{\mathbb{A}} \tilde{\mathbf{A}}$ such that

$$
\Delta\left(f \otimes \mathbf{k}_{c}\right)=\sum_{d=a+b} \Delta_{a, b}(f)\left(\mathbf{k}_{b+c} \otimes \mathbf{k}_{c}\right), \quad \forall f \in \mathbf{A}_{d}
$$

Then $(\tilde{\mathbf{A}}, \circ, \Delta)$ is a $\mathbb{A}$-bialgebra (it is due to Lusztig for the composition algebra, the general case is due to Green). Put $\tilde{\mathbf{U}}_{n}^{-}=\tilde{\mathbf{A}}$ if $\Gamma=\Gamma_{n}$ and $\tilde{\mathbf{U}}_{\infty}^{-}=\tilde{\mathbf{A}}$ if $\Gamma=\Gamma_{\infty}$. 
3.5. Given a $G_{V}$-orbit $O \subset E_{V}$ let $\mathbf{f}_{O} \in \mathbf{A}$ be the $v^{\operatorname{dim} O}$ times the characteristic function of $O$. For any $G_{V}$-orbit $O \subset E_{V}$ set

$$
\mathbf{b}_{O}=\sum_{i, O^{\prime}} v^{-i+\operatorname{dim} O-\operatorname{dim} O^{\prime}} \operatorname{dim} \mathcal{H}_{O^{\prime}}^{i}\left(I C_{O}\right) \mathbf{f}_{O^{\prime}} .
$$

The elements $\mathbf{b}_{O}$ form a basis of $\mathbf{A}$. If $d \in \mathbb{N}^{(I)}$ let $\mathbf{f}_{d} \in \mathbf{A}$ be the characteristic function of the zero representation of $\Gamma$ in a $d$-dimensional space. The following result is proved in Section 13.

Proposition. The algebra $\mathbf{A}$ is generated by the $\mathbf{f}_{d}, d \in \mathbb{N}^{(I)}$.

3.6. Given two integers $i \leq j$, let $\overline{\mathbb{F}}[i, j]$ be the unique indecomposable representation of $\Gamma_{\infty}\left(\right.$ resp. $\left.\Gamma_{n}\right)$ with dimension $\sum_{k=i}^{j} \epsilon_{k}$ (resp. $\left.\sum_{k=i}^{j} \epsilon_{\bar{k}}\right)$. For any partition $\lambda=\left(\lambda_{1} \geq \lambda_{2} \geq \cdots\right)$ let $\overline{\mathbb{F}}[\lambda]$ be the representation of $\Gamma$ such that

$$
\overline{\mathbb{F}}[\lambda]=\bigoplus_{k \geq 1} \overline{\mathbb{F}}\left[1-k, \lambda_{k}-k\right] .
$$

Let $O_{\lambda}$ be the orbit of $\overline{\mathbb{F}}[\lambda]$ and put $d_{\lambda}=\operatorname{dim} O_{\lambda}$.

\section{The Fock space.}

In this section we recall the construction of the quantized Fock space, due to $[\mathrm{H}]$, as it is re-interpreted in $[\mathrm{MM}]$.

4.1. Let $T(\lambda)$ be the tableau of shape $\lambda$ whose box with coordinates $(x, y)$ is filled with $y-x$. For instance if $\lambda=(432)$ we get

\begin{tabular}{|c|c|c|c}
\cline { 1 - 2 }-2 & -1 & \multicolumn{3}{|c}{} \\
\cline { 1 - 3 }-1 & 0 & 1 & \multicolumn{1}{|c}{} \\
\hline 0 & 1 & 2 & 3 \\
\hline
\end{tabular}

Let $\bigwedge^{\infty}$ be a $\mathbb{A}$-module with basis $\{|\lambda\rangle \mid \lambda \in \Pi\}$. If $i \in \mathbb{Z}$, a removable $i$-box of $T(\lambda)$ is a box with the color $i$ which can be removed in such a way that the new tableau still comes from a partition. Similarly, an indent $i$-box corresponds to a box with the color $i$ which can be added to $T(\lambda)$. Given $\bar{\imath} \in \mathbb{Z} / n \mathbb{Z}, i \in \bar{\imath}$, and a partition $\lambda$ put

$$
n_{i}(\lambda)=\sharp\{\text { indent } i \text {-box of } T(\lambda)\}-\sharp\{\text { removable } i \text {-box of } T(\lambda)\},
$$

and $n_{\bar{\imath}}(\lambda)=\sum_{i \in \bar{\imath}} n_{i}(\lambda), n_{i}^{-}(\lambda)=\sum_{j<i \& j \in \bar{\imath}} n_{j}(\lambda), n_{i}^{+}(\lambda)=\sum_{j>i \& j \in \bar{\imath}} n_{j}(\lambda)$.

4.2. The algebra $\mathbf{U}\left(\mathfrak{s l}_{\infty}\right)$ acts on $\bigwedge^{\infty}$ by

$$
\mathbf{k}_{i}(|\lambda\rangle)=v^{n_{i}(\lambda)}|\lambda\rangle, \quad \mathbf{e}_{i}(|\lambda\rangle)=|\nu\rangle, \quad \mathbf{f}_{i}(|\lambda\rangle)=|\mu\rangle,
$$

where the partitions $\mu, \nu$ are such that $T(\mu)-T(\lambda)$ and $T(\lambda)-T(\nu)$ are a box with color $i$. It is known that $\Lambda^{\infty}$ is the simple module with highest weight $\Lambda_{0}$ (the fundamental weight) and that the canonical basis of $\bigwedge^{\infty}$ is $\{|\lambda\rangle \mid \lambda \in \Pi\}$. The weight multiplicities in $\bigwedge^{\infty}$ are 0 or 1 , i.e. $\Lambda_{0}$ is a minuscule weight. 
4.3. The algebra $\mathbf{U}\left(\widehat{\mathfrak{s l}}_{n}\right)$ acts on $\bigwedge^{\infty}$ by

$$
\mathbf{k}_{\bar{\imath}}(|\lambda\rangle)=v^{n_{\bar{\imath}}(\lambda)}|\lambda\rangle, \quad \mathbf{e}_{\bar{\imath}}(|\lambda\rangle)=\sum_{i \in \bar{\imath}} v^{-n_{i}^{-}(\lambda)} \mathbf{e}_{i}(|\lambda\rangle), \quad \mathbf{f}_{\bar{\imath}}(|\lambda\rangle)=\sum_{i \in \bar{\imath}} v^{n_{i}^{+}(\lambda)} \mathbf{f}_{i}(|\lambda\rangle) .
$$

\section{The representation of $\mathbf{U}_{\infty}^{-}$on $\bigwedge^{\infty}$.}

The algebras $\mathbf{U}_{\infty}^{-}$and $\mathbf{U}^{-}\left(\mathfrak{s l}_{\infty}\right)$ are isomorphic. Thus $\bigwedge^{\infty}$ may be viewed as the quotient of $\mathbf{U}_{\infty}^{-}$by a left ideal I. Let us describe $\mathbf{I}$. Let $\bar{\Gamma}_{\infty}$ be the quiver $\Gamma_{\infty}$ with the opposit orientation. For any $\mathbb{Z}$-graded $\overline{\mathbb{F}}$-vector space $V$ let $\Lambda_{V}$ be the variety of pairs $(x, \bar{x})$ of commuting representations respectively of $\Gamma_{\infty}$ and $\bar{\Gamma}_{\infty}$ on $V$. The variety $\Lambda_{V}$ is reducible. For any $G_{V}$-orbit $O \subset E_{V}$ set

$$
\Lambda_{O}=\left\{(x, \bar{x}) \in \Lambda_{V} \mid x \in O\right\} .
$$

According to $[\mathrm{N}]$ the orbit $O$ is stable if there exists a triple

$$
(x, \bar{x}, i) \in \bar{\Lambda}_{O} \times \operatorname{Hom}(\overline{\mathbb{F}}[0], V)
$$

such that $i$ is homogeneous of degree 0 and that for any graded subspace $W \subseteq V$,

$$
(x(W), \bar{x}(W) \subseteq W \quad \text { and } \quad \operatorname{Im} i \subseteq W) \quad \Rightarrow \quad W=V
$$

(since the Hall algebra is endowed with the product opposit to the usual one, we use the stability condition opposit to the one in $[\mathrm{N}]$ ).

Proposition. The ideal $\mathbf{I}$ is linearly spanned by the elements $\mathbf{b}_{O}$ such that $O \neq O_{\lambda}$ for all $\lambda$. Moreover the map $\mathbf{U}_{\infty}^{-} / \mathbf{I} \rightarrow \bigwedge^{\infty}, \mathbf{b}_{O_{\lambda}}+\mathbf{I} \mapsto|\lambda\rangle$, is an isomorphism of $\mathbf{U}_{\infty}^{-}$-modules.

Proof. From [N, Theorem 11.7 and Proposition 3.5], $\mathbf{I}$ is linearly generated by the elements $\mathbf{b}_{O}$ such that $O$ is unstable. Let us show that for any $\lambda \in \Pi$ the orbit $O_{\lambda}$ is stable. A dimension counting then shows that the orbits $O_{\lambda}$ are precisely all the stable orbits. Recall that $\overline{\mathbb{F}}[i, j]$ is the representation $x$ of $\Gamma_{\infty}$ on the graded space $\bigoplus_{k=i}^{j} \overline{\mathbb{F}} v_{k}$, where $v_{k}$ is a non-zero vector of degree $k$, such that $x\left(v_{k}\right)=v_{k+1}$ if $k<j$ and $x\left(v_{j}\right)=0$. Fix $\lambda=\left(\lambda_{1}, \lambda_{2}, \ldots, \lambda_{r}\right) \in \Pi$. Fix non zero vectors $v_{k, s} \in$ $\overline{\mathbb{F}}\left[1-k, \lambda_{k}-k\right]$ with degree $s$. The representation $\overline{\mathbb{F}}[\lambda]$ is given by the endomorphism $x$ such that for all $k$,

$$
x\left(v_{k, s}\right)=v_{k, s+1} \quad \text { if } \quad s \in\left[1-k, \lambda_{k}-k\right), \quad \text { and } \quad x\left(v_{k, \lambda_{k}-k}\right)=0 .
$$

Let us exhibit a pair $(i, \bar{x})$ satisfying $(a)$. Fix a graded homomorphism $i \in \operatorname{Hom}(\overline{\mathbb{F}}[0], \overline{\mathbb{F}}[\lambda])$ such that $v_{1,0} \in \operatorname{Im} i$. Consider the degree -1 linear operator $\bar{x}$ on $\overline{\mathbb{F}}[\lambda]$ such that

$$
\bar{x}\left(v_{k, s}\right)=v_{k+1, s-1} \text { if } k \neq r \text { and } s \leq \lambda_{k+1}-k, \quad \bar{x}\left(v_{k, s}\right)=0 \text { else. }
$$

The operators $x$ and $\bar{x}$ commute since

$$
\begin{array}{ll}
x \bar{x}\left(v_{r, s}\right)=0=\bar{x} x\left(v_{r, s}\right) & \forall s, \\
x \bar{x}\left(v_{k, s}\right)=0=\bar{x} x\left(v_{k, s}\right) & \forall s \geq \lambda_{k+1}-k, \\
x \bar{x}\left(v_{k, s}\right)=v_{k+1, s}=\bar{x} x\left(v_{k, s}\right) & \forall s<\lambda_{k+1}-k, \forall k \neq r .
\end{array}
$$


Now if $W \subseteq V$ is such that $x(W) \subseteq W$ and $\operatorname{Im} i \subseteq W$ then $\overline{\mathbb{F}}\left[0, \lambda_{1}-1\right] \subseteq W$ : namely $v_{1,0} \in W$ and thus $v_{1, s}=x^{s}\left(v_{1,0}\right) \in W$ for all $s$. By definition of $\bar{x}$ we have for all $t<r$

$$
\bar{x}^{t}\left(\overline{\mathbb{F}}\left[0, \lambda_{1}-1\right]\right)=\overline{\mathbb{F}}\left[-t, \lambda_{1+t}-1\right] .
$$

The dimension $d_{\lambda}$ of $\overline{\mathbb{F}}[\lambda]$ is such that $d_{\lambda, i}$ is the multiplicity of the color $i$ in the tableau $T(\lambda)$ (see Section 4). Thus the linear isomorphism $\mathbf{b}_{O_{\lambda}} \mapsto|\lambda\rangle$ preserves the weights. Moreover it preserves the canonical base up to a permutation of its elements. Since $\Lambda_{0}$ is minuscule there is at most one vector of a given weight in the canonical basis. Hence the canonical bases are fully identified.

\section{The representation of $\mathbf{U}_{n}^{-}$on $\bigwedge^{\infty}$.}

6.1. Fix $d \in \mathbb{N}^{(\mathbb{Z})}$ and let $V$ be a $\mathbb{Z}$-graded $\overline{\mathbb{F}}$-vector space of dimension $d$. Let $\bar{d} \in \mathbb{N} \mathbb{Z} / n \mathbb{Z}$ be the multi-index such that $\bar{d}_{\bar{\imath}}=\sum_{j \in \bar{\imath}} d_{j}$ for all $\bar{\imath} \in \mathbb{Z} / n \mathbb{Z}$, and let $\bar{V}$ be the $\bar{d}$-dimensional $\mathbb{Z} / n \mathbb{Z}$-graded vector space such that $\bar{V}_{\bar{\imath}}=\bigoplus_{j \in \bar{\imath}} V_{j}$. The vector space $\bar{V}$ is filtered by the subspaces

$$
\bar{V}_{\geq i}=\bigoplus_{j \geq i} V_{j}, \quad \forall i \in \mathbb{Z}
$$

The associated graded is naturally identified with the $\mathbb{Z}$-graded space $V$. Set

$$
E_{\bar{V}, V}=\left\{x \in E_{\bar{V}} \mid x\left(\bar{V}_{\geq i}\right) \subseteq \bar{V}_{\geq i+1}, \quad \forall i\right\} .
$$

The map $p: E_{\bar{V}, V} \rightarrow E_{V}$ associate to a representation of $\Gamma_{n}$ in $\bar{V}$ the corresponding graded representation of $\Gamma_{\infty}$ in $V$. Let $j: E_{\bar{V}, V} \hookrightarrow E_{\bar{V}}$ be the closed embedding. Let consider the map $\gamma_{d}: \mathbf{U}_{n, \bar{d}}^{-} \rightarrow \mathbf{U}_{\infty, d}^{-}$such that

$$
\gamma_{d \mid v=q^{-1}}: \mathbb{C}_{G_{\bar{V}}}\left(E_{\bar{V}}\right) \rightarrow \mathbb{C}_{G_{V}}\left(E_{V}\right), \quad f \mapsto q^{-h(d)} p_{!} j^{*}(f),
$$

where $h(d)=\sum_{i<j \& \bar{\imath}=\bar{\jmath}} d_{i}\left(d_{j+1}-d_{j}\right)$. Put $k(b, a)=\sum_{i>j \& \bar{\imath}=\bar{\jmath}} b_{i}\left(2 a_{j}-a_{j-1}-a_{j+1}\right)$. The following is proved in Section 13.

Proposition. Fix $\alpha, \beta \in \mathbb{N}^{\mathbb{Z} / n \mathbb{Z}}$ and $d \in \mathbb{N}^{(\mathbb{Z})}$ such that $\bar{d}=\alpha+\beta$. Then,

$$
\sum_{\substack{a+b=d \\ \bar{a}=\alpha, \bar{b}=\beta}} v^{-k(b, a)} \gamma_{a}(f) \circ \gamma_{b}(g)=\gamma_{d}(f \circ g) \quad \forall f \in \mathbf{U}_{n, \alpha}^{-}, \forall g \in \mathbf{U}_{n, \beta}^{-},
$$

Remark. With the notations in Section 3.5 we have $\gamma_{d}\left(\mathbf{f}_{\bar{d}}\right)=v^{h(d)} \mathbf{f}_{d}$. Observe that $\mathbf{f}_{d}$ is the product of the divided powers $\mathbf{f}_{i}^{\left(d_{i}\right)}$, s ordered from $i=-\infty$ to $\infty$.

6.2. For all $\lambda \in \Pi$ and all $x \in \mathbf{U}_{n}^{-}$put

$$
x(|\lambda\rangle)=\sum_{d} \gamma_{d}(x) \mathbf{k}_{d^{\prime}}(|\lambda\rangle) \quad \text { where } \quad d^{\prime}=\sum_{j<i, \bar{\imath}=\bar{\jmath}} d_{j} \epsilon_{i} .
$$

Corollary. Formula (a) extends the Hayashi action of $\mathbf{U}^{-}\left(\widehat{\mathfrak{s l}}_{n}\right)$ on $\bigwedge^{\infty}$ to a representation of $\mathbf{U}_{n}^{-}$. 
Proof. The compatibility with the product in $\mathbf{U}_{n}^{-}$follows from Proposition 6.1. Formula (a) implies that

$$
\mathbf{f}_{\bar{\imath}}(|\lambda\rangle)=\sum_{i \in \bar{\imath}} \sum_{\mu} v^{g\left(\epsilon_{i}, d_{\lambda}\right)}|\mu\rangle
$$

where $\mu$ is a partition such that $T(\mu)-T(\lambda)$ is a box with color $i$ and

$$
g\left(\epsilon_{i}, d_{\lambda}\right)=-\sum_{\substack{i<j \\ \bar{j}=\bar{\imath}}}\left(2 d_{\lambda, j}-d_{\lambda, j-1}-d_{\lambda, j+1}\right)+\alpha_{i},
$$

where $\alpha_{i}=1$ if $i<0$ and $\bar{\imath}=0$, and $\alpha_{i}=0$ else. We have already observed that $d_{\lambda, i}$ is the multiplicity of the color $i$ in $T(\lambda)$. Thus, $n_{j}(\lambda)=-2 d_{\lambda, j}+d_{\lambda, j-1}+d_{\lambda, j+1}+\delta_{j 0}$, and

$$
g\left(\epsilon_{i}, d_{\lambda}\right)=\sum_{\substack{i<j \\ \bar{\jmath}=\bar{\imath}}} n_{j}(\lambda)=n_{i}^{+}(\lambda)
$$

6.3. For any $\lambda \in \Pi$ set $\mathbf{b}_{\lambda}=\mathbf{b}_{O_{\lambda}}|\emptyset\rangle$ where $O_{\lambda}$ is the isomorphism class of representations of $\Gamma_{n}$ defined in Subsection 3.6. Put $\mathbf{B}=\left\{\mathbf{b}_{\lambda} \mid \lambda \in \Pi\right\}$. Leclerc and Thibon have introduced in [LT] a semi-linear involution on $\bigwedge^{\infty}$.

Theorem. B is a basis of $\bigwedge^{\infty}$ whose elements are fixed by the Leclerc-Thibon involution.

The theorem is proved in Subsection 10.1. We first introduce some more material.

6.4. Let $r: E_{V} \rightarrow E_{\bar{V}}$ be such that

$$
r(x)_{\mid \bar{V}_{\bar{\imath}}}=\bigoplus_{i \in \bar{\imath}} x_{\mid V_{i}} \quad \forall x \in E_{V} .
$$

Fix a $G_{\bar{V}}$-orbit $O \subset E_{\bar{V}}$ such that $O \cap E_{\bar{V}, V} \neq \emptyset$. If $x \in p\left(O \cap E_{\bar{V}, V}\right)$ then

$$
\sharp\left(p^{-1}(x) \cap O\right) \in \begin{cases}q^{2 \mathbb{N}} & \text { if } r(x) \in O \\ \left(q^{2}-1\right) \mathbb{N} & \text { else. }\end{cases}
$$

Indeed, fix $y \in p^{-1}(x) \cap O$. It suffices to consider the case where $y$ is indecomposable. Then, fix a basis of homogeneous vectors $\left\{v_{i} \mid i \in[1, r]\right\}$ of $\bar{V}$ such that

$$
y\left(v_{k}\right)=v_{k+1} \quad \forall k=1,2, \ldots, r-1 .
$$

If $r(x) \in \bar{O} \backslash O$ then there exist $i, j$, such that $v_{i} \in \bar{V}_{\geq j} \backslash \bar{V}_{>j}$ and $v_{i+1} \in \bar{V}_{\geq j}$. If $t \in \mathbb{F}^{\times}$the representation $y_{t} \in E_{\bar{V}, V}$ obtained by doing $v_{k} \mapsto t v_{k}$ for all $k \leq i$ in $(c)$ is in $p^{-1}(x) \cap O$. Thus $\sharp\left(\mathbb{F}^{\times}\right) \mid \sharp\left(p^{-1}(x) \cap O\right)$. If $r(x) \in O$ then $r(x)$ and $y$ are isomorphic since $r(x), y \in O$. Thus $x$ is indecomposable and it is easy to see that $p^{-1}(x) \cap O$ is a vector space. We are done. The identity $(b)$ implies the following lemma which is used in Section 12.

Lemma. For any $G_{\bar{V}}$-orbit $O \subseteq E_{\bar{V}}$ we have

$$
\gamma_{d}\left(\mathbf{f}_{O}\right)=\mathbf{f}_{O^{\prime}} \bmod (v-1)
$$


where $O^{\prime} \subseteq E_{V}$ is the unique $G_{V}$-orbit such that $r\left(O^{\prime}\right) \subseteq O$.

\section{Flag varieties.}

7.1. Fix a positive integer $l$. Set $\mathbb{L}=\mathbb{F}((z))$ and $G=G L_{l}(\mathbb{L})$. A lattice in $\mathbb{L}^{l}$ is a free $\mathbb{F}[[z]]$-submodule of rank $l$. Let $Y$ be set of sequences of lattices $L=\left(L_{i}\right)_{i \in \mathbb{Z}}$ such that

$$
L_{i} \subseteq L_{i+1} \quad \text { and } \quad L_{i+n}=z^{-1} L_{i} .
$$

The group $G$ acts on $Y$ in the obvious way. Let $M$ be the set of all $\mathbb{Z} \times \mathbb{Z}$-matrices with non-negative entries, say $\mathbf{m}=\left(m_{i j}\right)_{i, j \in \mathbb{Z}}$, such that $m_{i+n, j+n}=m_{i j}$. Set

$$
M^{l}=\left\{\mathbf{m} \in M \mid \sum_{i \in \mathbb{Z}} \sum_{j=1}^{n} m_{i j}=l\right\}
$$

The set $M^{l}$ parametrizes the orbits of the diagonal action of $G$ in $Y \times Y$ : to $\mathbf{m}$ corresponds the set $Y_{\mathbf{m}}$ of the pairs $\left(L^{\prime}, L\right)$ such that

$$
m_{i j}=\operatorname{dim}_{\mathbb{F}}\left(\frac{L_{i+1} \cap L_{j+1}^{\prime}}{\left(L_{i} \cap L_{j+1}^{\prime}\right)+\left(L_{i+1} \cap L_{j}^{\prime}\right)}\right) .
$$

For all $L \in Y$ let $Y_{\mathbf{m}, L}$ be the fiber over $L$ of the first projection $Y_{\mathbf{m}} \rightarrow Y$. If $Y_{\mathbf{m}, L} \neq \emptyset$ then $Y_{\mathbf{m}, L}$ is the set of $\mathbb{F}$-points of an algebraic variety whose dimension, denoted by $y(\mathbf{m})$, is independent of $L$. Let $\mathbf{1}_{\mathbf{m}} \in \mathbb{C}_{G}(Y \times Y)$ be $q^{-y(\mathbf{m})}$ times the characteristic function of $Y_{\mathbf{m}}$. The convolution product, denoted $\star$, endows $\mathbb{C}_{G}(Y \times Y)$ with the structure of an associative algebra.

7.2. Let $X$ be the set of sequences of lattices $L=\left(L_{i}\right)_{i \in \mathbb{Z}}$ such that

$$
L_{i} \subseteq L_{i+1}, \quad L_{i+l}=z^{-1} L_{i} \quad \text { and } \quad \operatorname{dim}_{\mathbb{F}}\left(L_{i+1} / L_{i}\right)=1 .
$$

The group $G$ acts on $X$ in the obvious way. The orbits of the diagonal action of $G$ in $Y \times X$ are labelled by functions $\mathbf{i}: \mathbb{Z} \rightarrow \mathbb{Z}$ such that $\mathbf{i}(k+l)=\mathbf{i}(k)+n$ for all $k$ : let $X_{\mathbf{i}}$ be the orbit of the pair $\left(L_{\mathbf{i}}, L_{\emptyset}\right)$ such that

$$
L_{\mathbf{i}, i}=\prod_{\mathbf{i}(j) \leq i} \mathbb{F} e_{j} \quad \text { and } \quad L_{\emptyset, i}=\prod_{j \leq i} \mathbb{F} e_{j}
$$

Here $\left(e_{1}, e_{2}, \ldots, e_{l}\right)$ is a fixed $\mathbb{L}$-basis of $\mathbb{L}^{l}$ and $e_{i+l k}=z^{-k} e_{i}$ for all $k \in \mathbb{Z}$. A periodic function $\mathbf{i}$ as above is identified with the $l$-uple $(\mathbf{i}(1), \mathbf{i}(2), \ldots, \mathbf{i}(l)) \in \mathbb{Z}^{l}$. If $L \in Y$ let $X_{\mathbf{i}, L}$ be the fiber over $L$ of the projection $X_{\mathbf{i}} \rightarrow Y$. If $X_{\mathbf{i}, L} \neq \emptyset$, then $X_{\mathbf{i}, L}$ is the set of $\mathbb{F}$-points of an algebraic variety of dimension $l\left(\omega_{\mathbf{i}}\right)$. Let $\mathbf{1}_{\mathbf{i}} \in \mathbb{C}_{G}(Y \times X)$ be $q^{-l\left(\omega_{\mathbf{i}}\right)}$ times the characteristic function of $X_{\mathbf{i}}$. The space $\mathbb{C}_{G}(Y \times X)$ is a left $\mathbb{C}_{G}(Y \times Y)$-module and a right $\mathbb{C}_{G}(X \times X)$-module.

7.3. For all $x \in \widehat{\mathfrak{S}}_{l}$ let $X_{x} \subset X \times X$ be the $G$-orbit of the pair $\left(x\left(L_{\emptyset}\right), L_{\emptyset}\right)$. There is an algebras isomorphism $\widehat{\mathbf{H}}_{l \mid q^{-1}} \stackrel{\sim}{\rightarrow} \mathbb{C}_{G}(X \times X)$ which maps $T_{x}$ to the characteristic function of $X_{x}$ (see $\left.[\mathrm{IM}]\right)$. Put $P=X_{1}^{-1} \tilde{T}_{1}^{-1} \tilde{T}_{2}^{-1} \cdots \tilde{T}_{l-1}^{-1}$. 
Lemma. The right action of $\widehat{\mathbf{H}}_{l}$ on $\mathbb{C}_{G}(Y \times X)$ is such that if $\mathbf{i} \in A_{l}^{n}, x \in \mathfrak{S}^{\mathbf{i}}$, and $s \in \widehat{S}_{l}$, then $\left(\mathbf{1}_{\mathbf{i}}\right) P=\otimes \mathbf{1}_{(\mathbf{i}) \pi}$ and

$$
\left(\mathbf{1}_{(\mathbf{i}) x}\right) \tilde{T}_{s}= \begin{cases}v^{-1} \mathbf{1}_{(\mathbf{i}) x} & \text { if } x s \notin \mathfrak{S}^{\mathbf{i}} \quad(\text { then } \quad x s>x), \\ \mathbf{1}_{(\mathbf{i}) x s} & \text { if } x s>x \quad \text { and } \quad x s \in \mathfrak{S}^{\mathbf{i}}, \\ \mathbf{1}_{(\mathbf{i}) x s}+\left(v^{-1}-v\right) \mathbf{1}_{(\mathbf{i}) x} & \text { if } x s<x \quad\left(\text { then } \quad x s \in \mathfrak{S}^{\mathbf{i}}\right) .\end{cases}
$$

Proof. To simplify the notations fix $l=2$. Fix $\mathbf{i} \in A_{l}^{n}$ and $x \in \mathfrak{S}^{\mathbf{i}}$. Set $(i, j)=(\mathbf{i}) x$. Then

$$
x s_{1} \notin \mathfrak{S}^{\mathbf{i}} \Longleftrightarrow\left(\exists t \in S_{\mathbf{i}} \quad \text { such that } \quad x s_{1}=t x\right) \Longleftrightarrow(i, j) s_{1}=(i, j) .
$$

Moreover,

$$
x s_{1}>x \quad \text { and } \quad x s_{1} \in \mathfrak{S}^{\mathbf{i}} \Longleftrightarrow i \leq j \quad \text { and } \quad(i, j) s_{1} \neq(i, j) \text {. }
$$

The formulas in the proposition gives

$$
\left(\mathbf{1}_{(i, j)}\right) T_{1}= \begin{cases}v^{-2} \mathbf{1}_{(i, j)} & \text { if } i=j, \\ v^{-1} \mathbf{1}_{(j, i)} & \text { if } \quad i<j \\ v^{-1} \mathbf{1}_{(j, i)}+\left(v^{-2}-1\right) \mathbf{1}_{(i, j)} & \text { if } \quad i>j .\end{cases}
$$

These are precisely the formulas in [VV, Section 5] taking into account the different normalizations for the Hecke algebra and the factor $q^{-l\left(\omega_{\mathbf{i}}\right)}$. The result follows from [VV, Proposition 6].

7.4. Fix $\mathbf{i} \in A_{l}^{n}$. Let $\mathbf{H}_{\mathbf{i}} \subseteq \widehat{\mathbf{H}}_{l}$ be the parabolic subalgebra associated to $\mathfrak{S}_{\mathbf{i}}$. Set $e_{\mathbf{i}}=\sum_{x \in \mathfrak{S}_{\mathbf{i}}} T_{x}$ and $\pi_{\mathbf{i}}=\sum_{x \in \mathfrak{S}_{\mathbf{i}}} v^{-2 l(x)}$. Thus $e_{\mathbf{i}}^{2}=\pi_{\mathbf{i}} e_{\mathbf{i}}$ and $\bar{e}_{\mathbf{i}}=v^{2 l\left(\omega_{\mathbf{i}}\right)} e_{\mathbf{i}}$. Set

$$
\mathbf{T}_{n, l}=\bigoplus_{\mathbf{i} \in A_{l}^{n}} e_{\mathbf{i}} \widehat{\mathbf{H}}_{l} .
$$

The affine $q$-Schur algebra $\widehat{\mathbf{S}}_{n, l}$, introduced in [G2], is the endomorphism ring of the right $\widehat{\mathbf{H}}_{l}$-module $\mathbf{T}_{n, l}$. If $\mathbf{j} \in A_{l}^{n}$ set

$$
M_{\mathbf{i j}}=\left\{\mathbf{m} \in M^{l} \mid Y_{\mathbf{m}} \cap\left(G\left(L_{\mathbf{i}}\right) \times G\left(L_{\mathbf{j}}\right)\right) \neq \emptyset\right\} .
$$

A matrix $\mathbf{m} \in \mathbf{M}_{\mathbf{i j}}$ is identified with the class in $\mathfrak{S}_{\mathbf{i}} \backslash \widehat{\mathfrak{S}}_{l} / \mathfrak{S}_{\mathbf{j}}$ of the elements $x$ such that $\left(L_{(\mathbf{i}) x}, L_{\mathbf{j}}\right) \in Y_{\mathbf{m}}$. Set $T_{\mathbf{m}}=\sum_{x \in \mathbf{m}} T_{x}$. Let $\widehat{\mathbf{H}}_{\mathbf{i j}} \subseteq \widehat{\mathbf{H}}_{l}$ be the $\mathbb{A}$-linear span of the elements $T_{\mathbf{m}}$ with $\mathbf{m} \in M_{\mathbf{i j}}$. The $\mathbb{A}$-linear homomorphism

$$
\bigoplus_{\mathbf{i}, \mathbf{j} \in A_{l}^{n}} \widehat{\mathbf{H}}_{\mathbf{i j}} \rightarrow \widehat{\mathbf{S}}_{n, l}
$$


which maps $T_{\mathbf{m}}, \mathbf{m} \in M_{\mathbf{i j}}$, to the endomorphism such that $e_{\mathbf{k}} \mapsto \delta_{\mathbf{k}, \mathbf{j}} T_{\mathbf{m}} \in e_{\mathbf{i}} \widehat{\mathbf{H}}_{l}$, is invertible. The product in the affine $q$-Schur algebra, denoted $\bullet$, is

$$
T_{\mathbf{m}} \bullet T_{\mathbf{n}}=\delta_{\mathbf{k}, \mathbf{j}} \pi_{\mathbf{j}}^{-1} T_{\mathbf{m}} T_{\mathbf{n}} \quad \forall \mathbf{m} \in M_{\mathbf{i j}} \quad \forall \mathbf{n} \in M_{\mathbf{k l}} .
$$

If $t \in \mathbb{C}^{\times}$let $\widehat{\mathbf{S}}_{n, l \mid t}$ and $\mathbf{T}_{n, l \mid t}$ be the specializations of $\widehat{\mathbf{S}}_{n, l}$ and $\mathbf{T}_{n, l}$ at $v=t$.

Proposition. (a) The map $\Phi: \widehat{\mathbf{S}}_{n, l \mid q^{-1}} \rightarrow \mathbb{C}_{G}(Y \times Y), T_{\mathbf{m}} \mapsto q^{y(\mathbf{m})} \mathbf{1}_{\mathbf{m}}$, is an isomorphism of algebras.

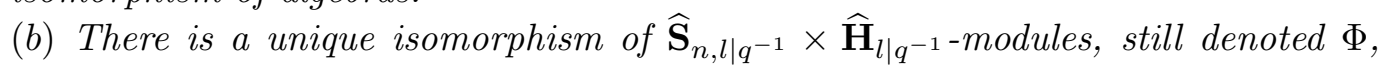
from $\mathbf{T}_{n, l \mid q^{-1}}$ to $\mathbb{C}_{G}(Y \times X)$ such that $e_{\mathbf{i}} \mapsto q^{l\left(\omega_{\mathbf{i}}\right)} \mathbf{1}_{\mathbf{i}}$ for all $\mathbf{i} \in A_{l}^{n}$.

Proof. The map $\Phi$ is a linear isomorphism. For all $x, y, z \in \widehat{\mathfrak{S}}_{l}$ let $B_{x y}^{z}(v) \in \mathbb{A}$ be such that

$$
T_{x} T_{y}=\sum_{z} B_{x y}^{z}(v) T_{z}
$$

If $\left(L^{\prime \prime}, L\right) \in X_{z}$ then,

$$
B_{x y}^{z}\left(q^{-1}\right)=\sharp\left\{L^{\prime} \in X \mid\left(L^{\prime \prime}, L^{\prime}\right) \in X_{x} \quad \& \quad\left(L^{\prime}, L\right) \in X_{y}\right\} .
$$

Fix $\mathbf{m} \in M_{\mathbf{i k}}, \mathbf{n} \in M_{\mathbf{i j}}$, and $\mathbf{o} \in M_{\mathbf{j k}}$. Let $A_{\mathbf{n o}}^{\mathbf{m}} \in \mathbb{N}$ be such that

$$
\mathbf{1}_{\mathbf{n}} \star \mathbf{1}_{\mathbf{o}}=\sum_{\mathbf{m}} q^{-y(\mathbf{n})-y(\mathbf{o})+y(\mathbf{m})} A_{\mathbf{n o}}^{\mathbf{m}} \mathbf{1}_{\mathbf{m}} .
$$

Then for any $z \in \mathbf{m}$,

$$
A_{\mathbf{n o}}^{\mathbf{m}}=h_{\mathbf{j}}^{-1} \sum_{\substack{x \in \mathbf{n} \\ y \in \mathbf{o}}} B_{x y}^{z}\left(q^{-1}\right),
$$

where $h_{\mathbf{j}}=\pi_{\mathbf{j} \mid v=q^{-1}}$ is the cardinal of the fiber of the projection $X \rightarrow G\left(L_{\mathbf{j}}\right)$. Claim (a) follows from the identity

$$
T_{\mathbf{n}} \bullet T_{\mathbf{o}}=\pi_{\mathbf{j}}^{-1} \sum_{z} \sum_{\substack{x \in \mathbf{n} \\ y \in \mathbf{o}}} B_{x y}^{z}(v) T_{z}=\sum_{\mathbf{m}} A_{\mathbf{n o}}^{\mathbf{m}} T_{\mathbf{m}} \quad \bmod \left(v-q^{-1}\right) .
$$

Let $\Phi$ be the unique isomorphism of right $\widehat{\mathbf{H}}_{l^{-} \text {modules }} \mathbf{T}_{n, l \mid q^{-1}} \stackrel{\sim}{\rightarrow} \mathbb{C}_{G}(Y \times X)$ such that $\Phi\left(e_{\mathbf{i}}\right)=q^{l\left(\omega_{\mathbf{i}}\right)} \mathbf{1}_{\mathbf{i}}$ for all $\mathbf{i} \in A_{l}^{n}$. Let us prove that $\Phi$ commutes to the action of $\widehat{\mathbf{S}}_{n, l}$. We must prove that for all $\mathbf{i}, \mathbf{j} \in A_{l}^{n}$ and all $\mathbf{m} \in M_{\mathbf{i j}}$ then

$$
q^{y(\mathbf{m})} \mathbf{1}_{\mathbf{m}} \star \mathbf{1}_{\mathbf{j}}=q^{-l\left(\omega_{\mathbf{j}}\right)} h_{\mathbf{i}}^{-1} \Phi\left(e_{\mathbf{i}} T_{\mathbf{m}}\right)=q^{l\left(\omega_{\mathbf{i}}\right)-l\left(\omega_{\mathbf{j}}\right)} h_{\mathbf{i}}^{-1}\left(\mathbf{1}_{\mathbf{i}}\right) T_{\mathbf{m}} .
$$

Put

$$
\mathbf{1}_{\mathbf{m}} \star \mathbf{1}_{\mathbf{j}}=\sum_{\mathbf{k} \in \mathbb{Z}^{l}} q^{-y(\mathbf{m})-l\left(\omega_{\mathbf{j}}\right)+l\left(\omega_{\mathbf{k}}\right)} A_{\mathbf{m} \mathbf{j}}^{\mathbf{k}} \mathbf{1}_{\mathbf{k}}, \quad\left(\mathbf{1}_{\mathbf{i}}\right) T_{\mathbf{m}}=\sum_{\mathbf{k} \in \mathbb{Z}^{l}} q^{-l\left(\omega_{\mathbf{i}}\right)+l\left(\omega_{\mathbf{k}}\right)} A_{\mathbf{i m}}^{\mathbf{k}} \mathbf{1}_{\mathbf{k}}
$$

Fix $z \in \widehat{\mathfrak{S}}_{l}$ and $\left(L^{\prime \prime}, L\right) \in X_{z}$ whose projection in $Y \times X$ is in $X_{\mathbf{k}}$. Then

$$
A_{\mathbf{m} \mathbf{j}}^{\mathbf{k}}=\sharp\left\{L^{\prime} \in Y \mid\left(L^{\prime \prime}, L^{\prime}\right) \in Y_{\mathbf{m}}, \quad\left(L^{\prime}, L\right) \in X_{\mathbf{j}}\right\}=h_{\mathbf{j}}^{-1} \sum_{\substack{y \in \mathbf{m} \\ x \in \mathfrak{S}_{\mathbf{j}}}} B_{y x}^{z}\left(q^{-1}\right),
$$




$$
A_{\mathrm{im}}^{\mathbf{k}}=\sum_{y \in \mathbf{m}} \sharp\left\{L^{\prime} \in X \mid\left(L^{\prime \prime}, L^{\prime}\right) \in X_{\mathbf{i}}, \quad\left(L^{\prime}, L\right) \in X_{y}\right\}=\sum_{\substack{y \in \mathbf{m} \\ x \in \mathfrak{S}_{\mathbf{i}}}} B_{x y}^{z}\left(q^{-1}\right) .
$$

Claim (b) follows from the equality

$$
\pi_{\mathbf{i}}^{-1} \sum_{\substack{y \in \mathfrak{m} \\ x \in \mathfrak{S}_{\mathbf{i}}}} T_{x} T_{y}=\pi_{\mathbf{j}}^{-1} \sum_{\substack{y \in \mathfrak{m} \\ x \in \mathfrak{S}_{\mathbf{j}}}} T_{y} T_{x}
$$

7.5. The set $M^{+}=\left\{\mathbf{m} \in M \mid i>j \Rightarrow m_{i j}=0\right\}$ parametrizes the isomorphism classes of nilpotent representations of the quiver $\Gamma_{n}: O_{\mathbf{m}}$ is the class of $\bigoplus_{i=1}^{n} \bigoplus_{j \geq i} \overline{\mathbb{F}}[i, j]^{m_{i j}}$. Let ${ }^{-}$be the unique semilinear involution on $\mathbf{U}_{n}^{-}$fixing the elements $\mathbf{b}_{O_{\mathrm{m}}}$.

Proposition. The involution ${ }^{-}$on $\mathbf{U}_{n}^{-}$is a ring homomorphism and $\overline{\mathbf{f}}_{\alpha}=\mathbf{f}_{\alpha}$ for all $\alpha \in \mathbb{N}^{(\mathbb{Z} / n \mathbb{Z})}$.

Proof. The second claim is obvious since $\mathbf{f}_{\alpha}$ is the characteristic function of a single point. We now prove the first claim. For any algebraic variety $X$ over $\overline{\mathbb{F}}$ let $\mathcal{D}(X)$ be the bounded derived category of complexes of $\mathbb{Q}_{l}$-sheaves on $X$ (see [L2], [L3]). If $G$ is a connected algebraic group acting on $X$ let $\mathcal{D}_{G}^{s s}(X)$ be the full subcategory whose objects are sums of shifted simple $G$-equivariant objects in $\mathcal{D}(X)$. Lusztig has defined in [L2, Section 3.1] a convolution product

$$
*: \mathcal{D}_{G_{U}}^{s s}\left(E_{U}\right) \times \mathcal{D}_{G_{W}}^{s s}\left(E_{W}\right) \rightarrow \mathcal{D}_{G_{V}}^{s s}\left(E_{V}\right)
$$

such that $\mathcal{F} * \mathcal{G}=\left(p_{3}\right)$ ! $\mathcal{H}$ where $\mathcal{H}$ satisfies $p_{2}^{*} \mathcal{H} \simeq p_{1}^{*}(\mathcal{F} \otimes \mathcal{G})$. Let $D$ be the Verdier duality. Since $p_{1}$ and $p_{2}$ are smooth with connected fibers and since $p_{3}$ is proper we get $D(\mathcal{F} * \mathcal{G})=(D \mathcal{F}) *(D \mathcal{G})\left[2 d_{1}-2 d_{2}\right]$ where $d_{1}$ and $d_{2}$ are the dimensions of the fibers of $p_{1}$ and $p_{2}$. Let $\alpha, \beta$ be the dimension of $U, W$. We know that $d_{2}=\sum_{\bar{\imath}} \alpha_{\bar{\imath}}^{2}+$ $\sum_{\bar{\imath}} \beta_{\bar{\imath}}^{2}$ and $d_{1}=d_{2}+m(\beta, \alpha)$. Thus $D(\mathcal{F} * \mathcal{G})=(D \mathcal{F}) *(D \mathcal{G})[2 m(\beta, \alpha)]$. Finally observe that the elements $\mathbf{b}_{O_{\mathrm{m}}}$ are the Frobenius traces of the simple perverse sheaves on the $E_{V}$ since the varieties $\bar{O}_{\mathbf{m}}$ are pure (see [L1, Corollary 11.6]).

7.6. If $L^{\prime}, L \in Y$ are such that $L^{\prime} \subseteq L$ then $L / L^{\prime}$ may be viewed as a nilpotent representation of $\Gamma_{n}$ of dimension $\alpha$ where $\alpha_{\bar{\imath}}=\operatorname{dim}_{\mathbb{F}}\left(L_{i} / L_{i}^{\prime}\right)$ (see [L2, Section 11], $[\mathrm{GV}])$. Then, set

$$
a\left(L^{\prime}, L\right)=\sum_{i=1}^{n} \operatorname{dim}_{\mathbb{F}}\left(L_{i} / L_{i}^{\prime}\right)\left(\operatorname{dim}_{\mathbb{F}}\left(L_{i+1}^{\prime} / L_{i}^{\prime}\right)-\operatorname{dim}_{\mathbb{F}}\left(L_{i} / L_{i}^{\prime}\right)\right) .
$$

Let $\Theta: \mathbf{U}_{n}^{-} \rightarrow \widehat{\mathbf{S}}_{n, l}$ be the $\mathbb{A}$-linear map such that

$$
\Phi \circ \Theta(f)\left(L^{\prime}, L\right)=q^{-a\left(L^{\prime}, L\right)} f\left(L / L^{\prime}\right) \quad \text { if } \quad L^{\prime} \subseteq L, \quad 0 \quad \text { else. }
$$

If $\mathbf{i} \in A_{l}^{n}$ and $\mathbf{m} \in M^{+}$let $\mathbf{m}^{\mathbf{i}} \in \cup_{\mathbf{j}} M_{\mathbf{i j}}$ be the matrix with the $(i, j)$-th entry equal to

$$
\delta_{i j}\left(\sharp \mathbf{i}^{-1}(j+1)-\sum_{k \leq j} m_{k j}\right)+\left(1-\delta_{i j}\right) m_{i+1, j} .
$$


Let $\phi$ be the semilinear involution on $\widehat{\mathbf{S}}_{n, l}$ such that $\phi(u)=v^{-2 l\left(\omega_{\mathbf{j}}\right)} \bar{u}$ for all $u \in \widehat{\mathbf{H}}_{\mathbf{i j}}$.

Proposition. The map $\Theta: \mathbf{U}_{n}^{-} \rightarrow \widehat{\mathbf{S}}_{n, l}$ is an algebra homomorphism. Moreover if $u \in \mathbf{U}_{n}^{-}$and $\mathbf{m} \in M^{+}$we have

$$
\phi \circ \Theta(u)=\Theta(\bar{u}) \quad \text { and } \quad \Phi \circ \Theta\left(\mathbf{f}_{O_{\mathbf{m}}}\right)=\sum_{\mathbf{i} \mid \mathbf{m}^{\mathbf{i}} \in M} \mathbf{1}_{\mathbf{m}^{\mathbf{i}}}
$$

Proof. The first claim is immediate from the formula

$$
a\left(L^{\prime \prime}, L\right)-a\left(L^{\prime}, L\right)-a\left(L^{\prime \prime}, L^{\prime}\right)=-m\left(L / L^{\prime}, L^{\prime} / L^{\prime \prime}\right)
$$

and from the definition of the product in $\mathbf{U}_{n}^{-}$and $\mathbb{C}_{G}(Y \times Y)$. We know that $\overline{\mathbf{f}}_{\alpha}=\mathbf{f}_{\alpha}$ for all $\alpha \in \mathbb{N}^{(\mathbb{Z} / n \mathbb{Z})}$. Similarly, $\phi \circ \Theta\left(\mathbf{f}_{\alpha}\right)=\Theta\left(\mathbf{f}_{\alpha}\right)$ since for any flag $L^{\prime}$ the $L^{\prime}$ 's such that $L^{\prime} \subseteq L$ and $\mathbf{f}_{\alpha}\left(L / L^{\prime}\right) \neq 0$ are the rational points of a smooth variety. Hence, the second claim results from the first claim, Proposition 3.5, and the fact that $\phi$ is a ring homomorphism. Now let us first prove that

(c) $\left(L^{\prime}, L\right) \in Y_{\mathbf{m}^{\mathrm{i}}} \quad \Longleftrightarrow \quad\left(L / L^{\prime} \in O_{\mathbf{m}} \quad\right.$ and $\left.\quad \operatorname{dim}_{\mathbb{F}}\left(L_{i}^{\prime} / L_{i-1}^{\prime}\right)=\sharp \mathbf{i}^{-1}(i)\right)$.

By definition $\left(L^{\prime}, L\right) \in Y_{\mathbf{m}^{\mathrm{i}}}$ if and only if

$$
\delta_{i j}\left(\sharp \mathbf{i}^{-1}(j+1)-\sum_{k \leq j} m_{k j}\right)+\left(1-\delta_{i j}\right) m_{i+1, j}=\operatorname{dim}_{\mathbb{F}}\left(\frac{L_{i+1} \cap L_{j+1}^{\prime}}{\left(L_{i} \cap L_{j+1}^{\prime}\right)+\left(L_{i+1} \cap L_{j}^{\prime}\right)}\right) .
$$

Thus it suffices to prove that if $\operatorname{dim}_{\mathbb{F}}\left(L_{i+1}^{\prime} / L_{i}^{\prime}\right)=\sharp \mathbf{i}^{-1}(i+1)$ and $L^{\prime} \subseteq L$ then

(d) $\operatorname{dim}_{\mathbb{F}}\left(\frac{L_{i} \cap L_{j+1}^{\prime}}{\left(L_{i-1} \cap L_{j+1}^{\prime}\right)+\left(L_{i} \cap L_{j}^{\prime}\right)}\right)$ is the multiplicity of $\mathbb{F}[i, j]$ in $L / L^{\prime}$ for all $i \leq j$,

(e) if $x_{\bar{\imath}}: L_{i} / L_{i}^{\prime} \rightarrow L_{i+1} / L_{i+1}^{\prime}$ is the map induced by the inclusion $L_{i} \subseteq L_{i+1}$, then

$$
\sharp \mathbf{i}^{-1}(i+1)-\operatorname{dim}_{\mathbb{F}} \operatorname{Ker}\left(x_{\bar{\imath}}\right)=\operatorname{dim}\left(L_{i+1}^{\prime} /\left(L_{i} \cap L_{i+1}^{\prime}\right)\right) .
$$

Claim $(e)$ is immediate since

$$
\operatorname{Ker}\left(x_{\bar{\imath}}\right)=\left(L_{i} \cap L_{i+1}^{\prime}\right) / L_{i}^{\prime}, \quad L_{i+1}^{\prime} /\left(L_{i} \cap L_{i+1}^{\prime}\right) \simeq \frac{L_{i+1}^{\prime} / L_{i}^{\prime}}{\left(L_{i} \cap L_{i+1}^{\prime}\right) / L_{i}^{\prime}},
$$

and since $\sharp \mathbf{i}^{-1}(i+1)=\operatorname{dim}\left(L_{i+1}^{\prime} / L_{i}^{\prime}\right)$. Part $(d)$ is due to the fact that $\mathbb{F}[i, j]$ is a direct summand of $L / L^{\prime}$ if and only if there is a vector $w \in L_{j+1}^{\prime} \backslash L_{j}^{\prime}$ such that $w \in L_{i} \backslash L_{i-1}$. The second claim follows from $(c)$ and the formula

$$
\left(\mathbf{m} \in M^{+} \quad \text { and } \quad \mathbf{m}^{\mathbf{i}} \in M\right) \Rightarrow \operatorname{dim} O_{\mathbf{m}}+a\left(\mathbf{m}^{\mathbf{i}}\right)=y\left(\mathbf{m}^{\mathbf{i}}\right),
$$

which is left to the reader.

Remark. For any $\mathbf{m} \in M^{l}$ set

$$
\mathbf{c}_{\mathbf{m}}=\sum_{i, \mathbf{n}} v^{-i+y(\mathbf{m})} \operatorname{dim} \mathcal{H}_{Y_{\mathbf{n}, L}}^{i}\left(I C_{Y_{\mathbf{m}, L}}\right) T_{\mathbf{n}},
$$


where $L$ is such that $Y_{\mathbf{m}, L} \neq \emptyset$. The elements $\mathbf{c}_{\mathbf{m}}$ form a $\mathbb{A}$-basis of $\widehat{\mathbf{S}}_{n, l}$ and $\phi\left(\mathbf{c}_{\mathbf{m}}\right)=\mathbf{c}_{\mathbf{m}}$. Proposition 7.6 implies that for any $\mathbf{m} \in M^{+}$we have

$$
\Theta\left(\mathbf{b}_{O_{\mathbf{m}}}\right)=\sum_{\mathbf{i} \mid \mathbf{m}^{\mathbf{i} \in M}} \mathbf{c}_{\mathbf{m}^{\mathbf{i}}}
$$

We will not use this.

\section{The tensor representation of $\tilde{\mathbf{U}}_{n}^{-}$.}

8.1. Let $\mathbb{A}^{(\mathbb{Z})}$ be the $\mathbb{A}$-linear span of vectors $\mathrm{x}_{i}, i \in \mathbb{Z}$. Let $\mathbf{e}_{i j} \in M$ be the matrix with 1 at the spot $(k, l)$ if $(k, l) \in(i, j)+\mathbb{Z}(n, n)$ and 0 elsewhere.

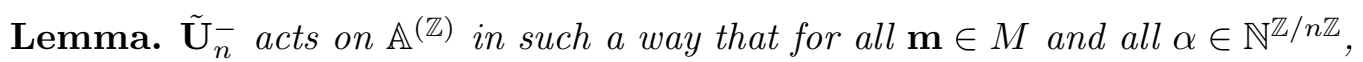

$$
\mathbf{f}_{O \mathbf{m}}\left(\mathrm{x}_{i}\right)=\sum_{j \geq i} \delta_{\mathbf{m}, \mathbf{e}_{i j}} \mathrm{x}_{j+1} \quad \text { and } \quad \mathbf{k}_{\alpha}\left(\mathrm{x}_{i}\right)=v^{-n\left(\alpha, \epsilon_{\bar{\imath}}\right)} \mathbf{x}_{i}
$$

Proof. It is the action obtained by taking $l=1$ in the geometric construction of Section 7 via the isomorphism $\mathbf{T}_{n, 1} \stackrel{\sim}{\rightarrow} \mathbb{A}(\mathbb{Z}), \mathbf{1}_{i} \mapsto \mathrm{x}_{i}$ (if $l=1$ then $X$ and $Y$ are zero dimensional).

8.2. Put $\bigotimes^{l}=\left(\mathbb{A}^{(\mathbb{Z})}\right)^{\otimes l}$. For any sequence $\mathbf{i}=\left(i_{1}, i_{2}, \ldots, i_{l}\right) \in \mathbb{Z}^{l}$ set $\otimes \mathrm{x}_{\mathbf{i}}=$ $\mathrm{x}_{i_{1}} \otimes \mathrm{x}_{i_{2}} \otimes \cdots \otimes \mathrm{x}_{i_{l}}$. On one hand $\otimes^{l}$ is a left $\tilde{\mathbf{U}}_{n}^{-}$-module via the coproduct $\Delta$. On the other hand $\widehat{\mathbf{H}}_{l}$ acts on $\otimes^{l}$ as follows for all $k=1,2, \ldots, l-1$ and $j=1,2, \ldots, l$ :

$(a)$

$$
\begin{aligned}
& \text { (a) }\left(\otimes \mathbf{x}_{\mathbf{i}}\right) T_{k}=\left\{\begin{array}{lll}
v^{-2} \otimes \mathrm{x}_{\mathbf{i}} & \text { if } & i_{k}=i_{k+1} \\
v^{-1} \otimes \mathrm{x}_{(\mathbf{i}) s_{k}} & \text { if } & -n<i_{k}<i_{k+1} \leq 0 \\
v^{-1} \otimes \mathrm{x}_{(\mathbf{i}) s_{k}}+\left(v^{-2}-1\right) \otimes \mathbf{x}_{\mathbf{i}} & \text { if } & -n<i_{k+1}<i_{k} \leq 0,
\end{array}\right. \\
& \text { (b) }\left(\otimes \mathrm{x}_{\mathbf{i}}\right) X_{j}^{-1}=\otimes \mathrm{x}_{(\mathbf{i}) \epsilon_{j} .}
\end{aligned}
$$

Lemma. The representations of $\tilde{\mathbf{U}}_{n}^{-}$and $\widehat{\mathbf{H}}_{l}$ on $\bigotimes^{l}$ commute.

Proof. Since the coproduct is coassociative (see [G1, Theorem 1(ii)]), we are reduced to the case $l=2$. By definition

$$
\Delta\left(\mathbf{f}_{\alpha}\right)=\sum_{\alpha=\beta+\gamma} v^{n(\gamma, \beta)} \mathbf{f}_{\beta} \mathbf{k}_{\gamma} \otimes \mathbf{f}_{\gamma} .
$$

Thus $\mathbf{f}_{\alpha}$ acts on $\bigotimes^{2}$ as

$$
\begin{cases}\mathbf{f}_{\bar{\imath}} \otimes 1+\mathbf{k}_{\bar{\imath}} \otimes \mathbf{f}_{\bar{\imath}} & \text { if } \quad \alpha=\epsilon_{\bar{\imath}} \\ \mathbf{f}_{\bar{\imath}} \otimes \mathbf{f}_{\bar{\jmath}}+\mathbf{f}_{\bar{\jmath}} \otimes \mathbf{f}_{\bar{\imath}} & \text { if } \quad \alpha=\epsilon_{\bar{\imath}}+\epsilon_{\bar{\jmath}} \quad \text { and } \quad \bar{\imath} \neq \bar{\jmath} \\ \mathbf{f}_{\bar{\imath}} \otimes \mathbf{f}_{\bar{\imath}} & \text { if } \alpha=2 \epsilon_{\bar{\imath}} \\ 0 & \text { else. }\end{cases}
$$


The commutation results from a direct computation.

8.3. Lemma. The map $\otimes \mathrm{x}_{\mathbf{i}} \mapsto v^{l\left(\omega_{\mathbf{i}}\right)} e_{\mathbf{i}}$, for all $\mathbf{i} \in A_{l}^{n}$, extends uniquely to an isomorphism of $\mathbf{U}_{n}^{-} \times \widehat{\mathbf{H}}_{l}$-bimodules $\otimes^{l} \stackrel{\sim}{\rightarrow} \mathbf{T}_{n, l}$.

Proof. The map above extends uniquely to an isomorphism of $\widehat{\mathbf{H}}_{l}$-modules. Let us prove that this isomorphism commutes to $\mathbf{U}_{n}^{-}$. For any $\mathbf{i} \in \mathbb{Z}^{l}$ Lemma 8.1 and formula $(c)$ give

$$
\mathbf{f}_{\alpha}\left(\otimes \mathbf{x}_{\mathbf{i}}\right)=\sum_{\mathbf{n}} v^{c(\mathbf{i}, \mathbf{i}+\mathbf{n})} \otimes \mathbf{x}_{\mathbf{i}+\mathbf{n}},
$$

where $\mathbf{n}=\left(n_{1}, n_{2}, \ldots, n_{l}\right) \in\{0,1\}^{l}$ describes the set of all sequences such that $\alpha=\sum_{s=1}^{l} n_{s} \epsilon_{\bar{\imath}_{s}}$ and

$$
c(\mathbf{i}, \mathbf{i}+\mathbf{n})=-\sum_{1 \leq s<t \leq l} n_{t}\left(1-n_{s}\right) n\left(\epsilon_{\bar{\imath}_{t}}, \epsilon_{\bar{\imath}_{s}}\right)
$$

By Proposition 7.4, after the specialisation $v=q^{-1}$ we have $q^{-l\left(\omega_{\mathbf{i}}\right)} \mathbf{f}_{\alpha}\left(e_{\mathbf{i}}\right)=(\Phi$ 。 $\Theta)\left(\mathbf{f}_{\alpha}\right) \star \mathbf{1}_{\mathbf{i}}$. The R.H.S. is the convolution product of $\mathbf{1}_{\mathbf{i}}$ and a function supported by the set of all pairs $\left(L^{\prime}, L\right)$ such that for all $i$ we have

(d) $\quad L_{i}^{\prime} \subseteq L_{i} \subseteq L_{i+1}^{\prime}, \quad \operatorname{dim}_{\mathbb{F}}\left(L_{i} / L_{i}^{\prime}\right)=\alpha_{i}, \quad$ and $\quad \operatorname{dim}_{\mathbb{F}}\left(L_{i} / L_{i-1}\right)=\sharp \mathbf{i}^{-1}(i)$

By definition of the convolution product $(\Phi \circ \Theta)\left(\mathbf{f}_{\alpha}\right) \star \mathbf{1}_{\mathbf{i}}\left(\operatorname{simply}\right.$ denoted by $\left.\mathbf{f}_{\alpha}\left(\mathbf{1}_{\mathbf{i}}\right)\right)$ is a linear combination of the $\mathbf{1}_{\mathbf{j}}$ 's such that it exists $L \in Y$ such that $\left(L, L_{\emptyset}\right) \in X_{\mathbf{i}}$ and $\left(L_{\mathbf{j}}, L\right)$ satisfies $(d)$. Suppose first that $\mathbf{i} \in A_{l}^{n}$. Then $X_{\mathbf{i}} \cap\left(Y \times\left\{L_{\emptyset}\right\}\right)=\left\{\left(L_{\mathbf{i}}, L_{\emptyset}\right)\right\}$. Thus $\mathbf{f}_{\alpha}\left(\mathbf{1}_{\mathbf{i}}\right)$ is a linear combination of the $\mathbf{1}_{\mathbf{j}}$ 's such that

$$
\mathbf{i} \leq \mathbf{j} \leq \mathbf{i}+1 \quad \text { and } \quad \alpha_{i}=\sharp\left(\mathbf{i}^{-1}(i) \cap \mathbf{j}^{-1}(i+1)\right) .
$$

More precisely we get

$$
\mathbf{f}_{\alpha}\left(\mathbf{1}_{\mathbf{i}}\right)=\sum_{\mathbf{n}} q^{-a(\mathbf{i}+\mathbf{n}, \mathbf{i})-l\left(\omega_{\mathbf{i}}\right)+l\left(\omega_{\mathbf{i}+\mathbf{n}}\right)} \mathbf{1}_{\mathbf{i}+\mathbf{n}},
$$

where the $\mathbf{n}$ 's are as above and $a(\mathbf{i}+\mathbf{n}, \mathbf{i})=\sum_{i} \alpha_{i}\left(\sharp \mathbf{i}^{-1}(i+1)-\alpha_{i+1}\right)$. A simple computation using $(e)$ gives

$$
a(\mathbf{i}+\mathbf{n}, \mathbf{i})=\sum_{s, t=1}^{l} n_{t}\left(1-n_{s}\right) \delta_{\bar{\imath}_{t}+1, \bar{\nu}_{s}}
$$

Moreover for any sequence $\mathbf{j}$ we have $l\left(\omega_{\mathbf{j}}\right)=\operatorname{dim}\left(P_{\mathbf{j}} /\left(B \cap P_{\mathbf{j}}\right)\right)$ where $P_{\mathbf{j}}, B \subset G$ are the isotropy subgroup of $L_{\mathbf{j}}$ and $L_{\emptyset}$. Thus we obtain

$$
\begin{aligned}
& l\left(\omega_{\mathbf{i}+\mathbf{n}}\right)-l\left(\omega_{\mathbf{i}}\right)=\sum_{1 \leq t<s \leq l} n_{t}\left(1-n_{s}\right) \delta_{\bar{l}_{s}, \bar{\nu}_{t}+1}\left(1-\delta_{i_{t}, 0}\right)+ \\
& +\sum_{1 \leq t<s \leq l} n_{s}\left(1-n_{t}\right) \delta_{i_{t}, 1-n} \delta_{i_{s}, 0}-\sum_{1 \leq t<s \leq l} n_{s}\left(1-n_{t}\right) \delta_{\bar{\imath}_{t}, \bar{\nu}_{s}} .
\end{aligned}
$$


To conclude it suffices to compute the image of $\otimes \mathrm{x}_{\mathbf{i}+\mathbf{n}}$ in $\mathbb{C}_{G}(Y \times X)$. Using the identity

$$
X_{k}^{-1}=\tilde{T}_{k-1}^{-1} \cdots \tilde{T}_{1}^{-1} P \tilde{T}_{l-1} \cdots \tilde{T}_{k}
$$

and Lemmas 7.3 and 8.2, we get that $\otimes \mathrm{x}_{\mathbf{i}+\mathbf{n}}$ is mapped to

$$
q^{-d(\mathbf{i}, \mathbf{i}+\mathbf{n})} \mathbf{1}_{\mathbf{i}+\mathbf{n}}, \quad d(\mathbf{i}, \mathbf{i}+\mathbf{n})=\sharp\left\{1 \leq s, t \leq l \mid i_{s}=0, i_{t}=1-n, n_{s}=1, n_{t}=0\right\} .
$$

Then, the equality results from an easy computation. The general case (i.e. $\mathbf{i} \notin A_{l}^{n}$ ) follows since the isomorphism we consider commutes to $\widehat{\mathbf{H}}_{l}$.

8.4. Let $\psi$ be the semilinear involution on $\mathbf{T}_{n, l} \simeq \bigotimes^{l}$ such that $\psi\left(e_{\mathbf{i}} t\right)=\bar{e}_{\mathbf{i}} \bar{t}$ for all $t \in \widehat{\mathbf{H}}_{l}$. Proposition 7.6 and the definition of the involutions $\phi$ and $\psi$ imply the following lemma (see Subsection 7.6).

Lemma. For all $u \in \mathbf{U}_{n}^{-}$and all $t \in \bigotimes^{l}$ we have $\psi(u t)=\bar{u} \psi(t)$.

\section{The action of $\mathrm{U}_{n}^{-}$on wedges.}

9.1. Set $\Omega^{l}=\sum_{i} \operatorname{Im}\left(T_{i}+1\right) \subset \bigotimes^{l}$. We have

$$
\bigotimes^{l} / \Omega^{l} \simeq \bigoplus_{\mathbf{i} \in A_{l}^{n}} e_{\mathbf{i}} \widehat{\mathbf{H}}_{l} e^{-},
$$

where $e^{-}=\sum_{x \in \mathfrak{S}_{l}}(-v)^{l(x)} \tilde{T}_{x}$. For any $\mathbf{i} \in \mathbb{Z}^{l}$ let $\wedge \mathrm{x}_{\mathbf{i}}$ be the image of $\otimes \mathrm{x}_{\mathbf{i}}$ in $\otimes^{l} / \Omega^{l}$. Set

$$
P_{l}^{++}=\left\{\mathbf{i} \in \mathbb{Z}^{l} \mid i_{1}>i_{2}>\ldots>i_{l}\right\} .
$$

The $\wedge \mathrm{x}_{\mathbf{i}}$ 's such that $\mathbf{i} \in P_{l}^{++}$form a basis of $\bigotimes^{l} / \Omega^{l}$ (see [KMS, Proposition 1.3]). For any $\lambda \in \Pi_{l}$ set $|\lambda\rangle=\wedge \mathbf{x}_{\mathbf{i}}$ if $\mathbf{i}=\lambda+\rho$, where $\rho$ is as in Section 1.1. Let $\Lambda^{l} \subset \bigotimes^{l} / \Omega^{l}$ be the linear span of the vectors $|\lambda\rangle$.

9.2. The representation of $\mathbf{U}_{n}^{-}$on $\bigotimes^{l}$ descends to $\bigotimes^{l} / \Omega^{l}$ (use Lemma 8.2). For all $\lambda \in \Pi_{l}$ set $\mathbf{b}_{\lambda}=\mathbf{b}_{O_{\lambda}}|\emptyset\rangle$ and put $\mathbf{B}_{l}=\left\{\mathbf{b}_{\lambda} \mid \lambda \in \Pi_{l}\right\}$. Let consider the involution $\psi$ on $\otimes^{l} / \Omega^{l}$ such that

$$
\psi\left(e_{\mathbf{i}} h e^{-}\right)=v^{2 l(\omega)} \bar{e}_{\mathbf{i}} \bar{h} e^{-} \quad \forall h \in \widehat{\mathbf{H}}_{l} .
$$

Proposition. $\mathbf{B}_{l}$ is a basis of $\bigwedge^{l}$ whose elements are fixed by $\psi$.

Proof. Lemma 8.1, the definition of $\Delta$, and the normal ordering rule [KMS, (43) and (45)] imply that for any $\lambda \in \Pi_{l}$ and any orbit $O \subset \bar{O}_{\lambda} \backslash O_{\lambda}$ we have

$$
\mathbf{f}_{O_{\lambda}}(|\emptyset\rangle) \in v^{\mathbb{Z}}|\lambda\rangle+\bigoplus_{\mu<\lambda} \mathbb{A}|\mu\rangle \quad \text { and } \quad \mathbf{f}_{O}(|\emptyset\rangle) \in \bigoplus_{\mu<\lambda} \mathbb{A}|\mu\rangle .
$$

Thus, $\mathbf{B}_{l}$ is a basis. Now Lemma 8.4 implies that the action of $\mathbf{b}_{O_{\mathbf{m}}}$ on $\bigwedge^{l}$ commutes to $\psi$. Since $\psi(|\emptyset\rangle)=|\emptyset\rangle$ we get $\psi\left(\mathbf{b}_{\lambda}\right)=\mathbf{b}_{\lambda}$ for all $\lambda$.

9.3. Let $P_{l}^{+} \subset \mathbb{Z}^{l}$ be the subset of integral dominant weights. Let $\mathfrak{S}^{\mathbf{i}, l}$ be the set of minimal length representatives of the cosets in $\mathfrak{S}_{\mathbf{i}} \backslash \widehat{\mathfrak{S}}_{l} / \mathfrak{S}_{l}$. Thus $\mathfrak{S}^{\mathbf{i}, l}=\mathfrak{S}^{\mathbf{i}} \cap \mathfrak{S}^{l}$ 
where $\mathfrak{S}^{l}$ is the set of minimal length representatives of the cosets in $\widehat{\mathfrak{S}}_{l} / \mathfrak{S}_{l}$. For any $x \in \widehat{\mathfrak{S}}_{l}$ let $\check{x}$ be the smallest element in the double coset $\mathfrak{S}_{\mathbf{i}} x \mathfrak{S}_{l}$. Set

$$
\mathfrak{S}(\mathbf{i}, l)=\left\{x \in \mathfrak{S}^{\mathbf{i}, l} \mid S_{\mathbf{i}} x \cap x S_{l}=\emptyset\right\}
$$

Lemma. Fix $\mathbf{i} \in A_{l}^{n}$. Then, (a) $e_{\mathbf{i}} \tilde{T}_{x} e^{-} \neq 0 \Rightarrow x \in \mathfrak{S}_{\mathbf{i}} \mathfrak{S}(\mathbf{i}, l) \mathfrak{S}_{l}$,

(b) $\quad\left(x \in \mathfrak{S}^{\mathbf{i}} \quad \& \quad(\mathbf{i}) x \in P_{l}^{++}\right) \Rightarrow e_{\mathbf{i}} \tilde{T}_{x} e^{-}=v^{-l\left(\omega_{\mathbf{i}}\right)} \wedge \mathbf{x}_{(\mathbf{i}) x}$,

(c) $\quad(\mathbf{i}) x \in P_{l}^{++} \Longleftrightarrow x \in \mathfrak{S}_{\mathbf{i}} \mathfrak{S}(\mathbf{i}, l) \omega$.

Proof. Suppose that $x \in \mathfrak{S}^{\mathbf{i}, l}, s_{i} \in S_{\mathbf{i}}$, and $s \in S_{l}$, are such that $s_{i} x=x s$. Then

$$
v^{-1} e_{\mathbf{i}} \tilde{T}_{x} e^{-}=e_{\mathbf{i}} \tilde{T}_{s_{i}} \tilde{T}_{x} e^{-}=e_{\mathbf{i}} \tilde{T}_{x} \tilde{T}_{s} e^{-}=-v e_{\mathbf{i}} \tilde{T}_{x} e^{-} .
$$

Thus $e_{\mathbf{i}} \tilde{T}_{x} e^{-}=0$. Any $x \in \widehat{\mathfrak{S}}_{l}$ decomposes in $x=x_{\mathbf{i}} \check{x} x_{l}$ where $x_{\mathbf{i}} \in \mathfrak{S}_{\mathbf{i}}, \check{x} \in \mathfrak{S}^{\mathbf{i}, l}$, $x_{l} \in \mathfrak{S}_{l}$, and $l(x)=l\left(x_{\mathbf{i}}\right)+l(\check{x})+l\left(x_{l}\right)$. In particular

$$
e_{\mathbf{i}} \tilde{T}_{x} e^{-}=v^{-l\left(x_{\mathbf{i}}\right)}(-v)^{l\left(x_{l}\right)} e_{\mathbf{i}} \tilde{T}_{\breve{x}} e^{-} .
$$

Claim (a) follows. Let us prove claim $(b)$. Recall that if $\lambda$ is dominant then $\tilde{T}_{\lambda}^{-1}$ is mapped to $X^{\lambda}=X_{1}^{\lambda_{1}} X_{2}^{\lambda_{2}} \cdots X_{l}^{\lambda_{l}}$ by the Bernstein isomorphism. Then (8.2.b) implies that

$$
\left(\otimes \mathrm{x}_{\mathbf{i}}\right) \tilde{T}_{\lambda}=\otimes \mathrm{x}_{(\mathbf{i}) \lambda} \quad \forall \lambda \in P_{l}^{+} \quad \forall \mathbf{i} \in \mathbb{Z}^{l},
$$

Moreover (8.2.a) implies that

$$
\left(\otimes \mathrm{x}_{\mathbf{i}}\right) \tilde{T}_{x}=\otimes \mathrm{x}_{(\mathbf{i}) x} \quad \forall x \in \mathfrak{S}_{l} \cap \mathfrak{S}^{\mathbf{i}} \quad \forall \mathbf{i} \in A_{l}^{n}
$$

Fix $x \in \widehat{\mathfrak{S}}_{l}$. Then $x$ decomposes uniquely as $x=y \lambda$ where $y \in \mathfrak{S}_{l}$ and $\lambda \in \mathbb{Z}^{l}$. If (i) $x=(\mathbf{i}) y+n \lambda \in P_{l}^{++}$then $\lambda \in P_{l}^{+}$. Since $s \lambda>\lambda$ for all $s \in S_{l}$ if $\lambda$ is dominant, we get $\tilde{T}_{x}=\tilde{T}_{y} \tilde{T}_{\lambda}$. Suppose moreover that $x \in \mathfrak{S}^{\mathbf{i}}$. Then for any $s \in S_{\mathbf{i}}$ we have sy $\lambda>y \lambda$. Since $l(z \lambda)=l(z)+l(\lambda)$ for any $z \in \mathfrak{S}_{l}$ ( $\lambda$ is dominant $)$, we obtain that $y \in \mathfrak{S}_{l} \cap \mathfrak{S}^{\mathbf{i}}$. Hence, Section 8.2 implies that

$$
e_{\mathbf{i}} \tilde{T}_{x} e^{-}=e_{\mathbf{i}} \tilde{T}_{y} \tilde{T}_{\lambda} e^{-}=v^{-l\left(\omega_{\mathbf{i}}\right)} \wedge \mathrm{x}_{(\mathbf{i}) x} .
$$

Finally, claim $(c)$ follows from

$$
\begin{aligned}
\left(x \in \mathfrak{S}^{\mathbf{i}} \quad \& \quad(\mathbf{i}) x \in P_{l}^{++}\right) & \Longleftrightarrow\left(s_{i} x>x>x s \quad \forall s \in S_{l} \quad \forall s_{i} \in S_{\mathbf{i}}\right) \\
& \Longleftrightarrow x \in \mathfrak{S}(\mathbf{i}, l) \omega .
\end{aligned}
$$

Proposition. If $\mathbf{i} \in A_{l}^{n}$ and $(\mathbf{i}) x \in P_{l}^{++}$then $\psi\left(\wedge \mathrm{x}_{(\mathbf{i}) x}\right)=(-1)^{l(\omega)} v^{l\left(\omega^{\mathbf{i}}\right)} \wedge \mathrm{x}_{(\mathbf{i}) x \omega}$.

Proof. First recall that if $\lambda \in P_{l}^{+}$and $\lambda^{*}=-\omega(\lambda)$, then $\tilde{T}_{\lambda^{*}}=\tilde{T}_{\omega}^{-1} \tilde{T}_{-\lambda} \tilde{T}_{\omega}$ (indeed, since $\lambda, \lambda^{*}$ are dominant weights we have $\tilde{T}_{\omega} \tilde{T}_{\lambda^{*}}=\tilde{T}_{-\lambda \omega}=\tilde{T}_{-\lambda} \tilde{T}_{\omega}$. In particular, 
$\left.\tilde{T}_{-\lambda}=\tilde{T}_{\omega} \tilde{T}_{\lambda^{*}} \tilde{T}_{\omega}^{-1}\right)$. Fix $\mathbf{i} \in A_{l}^{n}$ and $x \in \mathfrak{S}^{\mathbf{i}}$ such that $(\mathbf{i}) x \in P_{l}^{++}$. As above fix $x=y \lambda$ with $y \in \mathfrak{S}_{l} \cap \mathfrak{S}^{\mathbf{i}}$ and $\lambda \in P_{l}^{+}$. Using Lemma 9.3.b we get

$$
\begin{gathered}
\psi\left(\wedge \mathrm{x}_{(\mathbf{i}) x}\right)=\psi\left(\left(\otimes \mathrm{x}_{\mathbf{i}}\right) \tilde{T}_{y} \tilde{T}_{\lambda} e^{-}\right)=v^{-l\left(\omega_{\mathbf{i}}\right)} \psi\left(e_{\mathbf{i}} \tilde{T}_{y} \tilde{T}_{\lambda} e^{-}\right)=v^{l\left(\omega_{\mathbf{i}}\right)+2 l(\omega)} e_{\mathbf{i}} \tilde{T}_{y^{-1}}^{-1} \tilde{T}_{-\lambda}^{-1} e^{-}= \\
=v^{l\left(\omega_{\mathbf{i}}\right)+2 l(\omega)} e_{\mathbf{i}} \tilde{T}_{y^{-1}}^{-1} \tilde{T}_{\omega} \tilde{T}_{\lambda^{*}}^{-1} \tilde{T}_{\omega}^{-1} e^{-}=(-1)^{l(\omega)} v^{2 l(\omega)-l\left(\omega^{\mathbf{i}}\right)} e_{\mathbf{i}} \tilde{T}_{y \omega} \tilde{T}_{\lambda^{*}}^{-1} e^{-} .
\end{gathered}
$$

For all $s \in S_{\mathbf{i}}, s y>y$ implies that $s y \omega<y \omega$. Thus

$$
\psi\left(\wedge \mathrm{x}_{(\mathbf{i}) x}\right)=(-1)^{l(\omega)} v^{l\left(\omega^{\mathbf{i}}\right)}\left(\otimes \mathrm{x}_{(\mathbf{i}) y \omega}\right) \tilde{T}_{\lambda^{*}}^{-1} e^{-}=(-1)^{l(\omega)} v^{l\left(\omega^{\mathbf{i}}\right)} \wedge \mathrm{x}_{(\mathbf{i}) x \omega} .
$$

9.4. Fix $x \in \mathfrak{S}^{l}$. The element

$$
D_{x \omega}=\sum_{\substack{y \in \mathfrak{S}^{l} \\ y \leq x}}(-v)^{l(y)-l(x)} \bar{P}_{y \omega, x \omega} \tilde{T}_{y \omega} e^{-}
$$

is fixed by the involution on $\widehat{\mathbf{H}}_{l} e^{-}$such that $h e^{-} \mapsto v^{2 l(\omega)} \bar{h} e^{-}$and

$$
D_{x \omega}-\tilde{T}_{x \omega} e^{-} \in \bigoplus_{y \in \mathfrak{S}_{l}} v^{-1} \overline{\mathbb{S}} \tilde{T}_{y \omega} e^{-}
$$

(see [D1]). If $\mathbf{i} \in A_{l}^{n}$ and $x \in \mathfrak{S}(\mathbf{i}, l)$ set $\mathbf{b}_{(\mathbf{i}) x \omega}^{-}=v^{l\left(\omega_{\mathbf{i}}\right)} e_{\mathbf{i}} D_{x \omega}$. Lemma 9.3 gives

$$
\mathbf{b}_{(\mathbf{i}) x \omega}^{-}=\sum_{\substack{y \in \mathfrak{S}^{l} \\ y \leq x}}(-v)^{l(y)-l(x)} v^{-l\left(y_{\mathbf{i}}\right)} \bar{P}_{y \omega, x \omega} \wedge \mathrm{x}_{(\mathbf{i}) y \omega} .
$$

Similarly fix $\mathbf{i} \in A_{l}^{n}$ and $x \in \mathfrak{S}^{\mathbf{i}}$. Then

$$
C_{\omega_{\mathbf{i}} x}^{\prime}=v^{l(x)+l\left(\omega_{\mathbf{i}}\right)} \sum_{\substack{y \in \mathfrak{S}^{\mathbf{i}} \\ y \leq x}} \sum_{z \in \mathfrak{S}_{\mathbf{i}}} P_{z y, \omega_{\mathbf{i}} x} T_{z y}
$$

If $y \in \mathfrak{S}^{\mathbf{i}}$ and $y \leq x$ then $P_{z y, \omega_{\mathbf{i}} x}=P_{\omega_{\mathbf{i}} y, \omega_{\mathbf{i}} x}$ for all $z \in \mathfrak{S}_{\mathbf{i}}$ (see [D1, page 491]). Thus

$$
C_{\omega_{\mathbf{i}} x}^{\prime}=v^{l(x)+l\left(\omega_{\mathbf{i}}\right)} e_{\mathbf{i}} \sum_{\substack{y \in \mathfrak{S}^{\mathbf{i}} \\ y \leq x}} P_{\omega_{\mathbf{i}} y, \omega_{\mathbf{i}} x} T_{y}
$$

If $x \in \mathfrak{S}(\mathbf{i}, l)$ set $\mathbf{b}_{(\mathbf{i}) x \omega}^{+}=(-v)^{l(\omega)} C_{\omega_{\mathbf{i} x}}^{\prime} e^{-}$. Then Lemma 2.2 gives

$$
\begin{aligned}
\mathbf{b}_{(\mathbf{i}) x \omega}^{+} & =\sum_{(y, z)} v^{l(x)-l(y z)}(-v)^{l(z)} P_{\omega_{\mathbf{i}} y z, \omega_{\mathbf{i}} x} \wedge \mathrm{x}_{(\mathbf{i}) y \omega} \\
& =\sum_{\substack{y \in \mathfrak{S}(\mathbf{i}, l) \\
y \leq x}} v^{l(x)-l(y)} Q_{\omega_{\mathbf{i}} y, \omega_{\mathbf{i}} x} \wedge \mathrm{x}_{(\mathbf{i}) y \omega},
\end{aligned}
$$

where the first sum is over all couples $(y, z) \in \mathfrak{S}(\mathbf{i}, l) \times \mathfrak{S}_{l}$ such that $y z \leq x$ and $Q_{\omega_{\mathbf{i}} y, \omega_{\mathbf{i}} x}=\sum_{z}(-1)^{l(z)} P_{\omega_{\mathbf{i}} y z, \omega_{\mathbf{i}} x}$ is a parabolic Kazhdan-Lusztig polynomial. Observe that $\mathbf{b}_{(\mathbf{i}) x \omega}^{ \pm}$is completely characterized by the following properties :

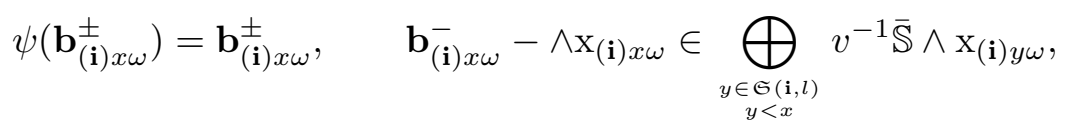




$$
\text { and } \quad \mathbf{b}_{(\mathbf{i}) x \omega}^{+}-\wedge \mathrm{x}_{(\mathbf{i}) x \omega} \in \bigoplus_{\substack{y \in \mathfrak{S}(\mathbf{i}, l) \\ y<x}} v \mathbb{S} \wedge \mathrm{x}_{(\mathbf{i}) y \omega} .
$$

In particular $\left\{\mathbf{b}_{\mathbf{i}}^{-} \mid \mathbf{i} \in P_{l}^{++}\right\}$and $\left\{\mathbf{b}_{\mathbf{i}}^{+} \mid \mathbf{i} \in P_{l}^{++}\right\}$are bases of $\bigotimes^{l} / \Omega^{l}$. For all $\lambda \in \Pi_{l}$ set $\mathbf{b}_{\lambda}^{ \pm}=\mathbf{b}_{\mathbf{i}}^{ \pm}$if $\mathbf{i}=\lambda+\rho$. Put $\mathbf{B}_{l}^{ \pm}=\left\{\mathbf{b}_{\lambda}^{ \pm} \mid \lambda \in \Pi_{l}\right\}$.

Remark. If $\mathbf{i} \in A_{l}^{n}$ and $x, y \in \mathfrak{S}^{\mathbf{i}}$ are such that if $(\mathbf{i}) x,(\mathbf{i}) y \in P_{l}^{++}$then

$$
y \leq x \Rightarrow(\mathbf{i}) x-(\mathbf{i}) y \text { is a positive root. }
$$

9.5. The space $\bigwedge^{l}$ is endowed with four bases : $\mathbf{B}_{l}^{ \pm}=\left\{\mathbf{b}_{\lambda}^{ \pm} \mid \lambda \in \Pi_{l}\right\}, \mathbf{B}_{l}=\left\{\mathbf{b}_{\lambda} \mid \lambda \in\right.$ $\left.\Pi_{l}\right\}$, and $\left\{|\lambda\rangle \mid \lambda \in \Pi_{l}\right\}$. Moreover, $\mathbf{B}_{l}^{ \pm}$are characterized by

$$
\psi\left(\mathbf{b}_{\lambda}^{ \pm}\right)=\mathbf{b}_{\lambda}^{ \pm}, \quad \mathbf{b}_{\lambda}^{-}-|\lambda\rangle \in \bigoplus_{\mu<\lambda} v^{-1} \overline{\mathbb{S}}|\mu\rangle \quad \text { and } \quad \mathbf{b}_{\lambda}^{+}-|\lambda\rangle \in \bigoplus_{\mu<\lambda} v \mathbb{S}|\mu\rangle
$$

(in particular $\left.\psi\left(\bigwedge^{l}\right)=\bigwedge^{l}\right)$. Recall that if $x \in \widehat{\mathfrak{S}}_{l}$ and $\lambda \in \mathbb{Z}^{l}$, then $\lambda \cdot x=(\lambda+\rho) x-\rho$ (see 1.1). Section 9.4 implies the following theorem.

Theorem. (a) If $\lambda \in \Pi_{l}$ and $x$ is minimal such that $\mathbf{i}=\lambda \cdot x^{-1}+\rho \in A_{l}^{n}$, then

$$
\mathbf{b}_{\lambda}^{-}=\sum_{y}(-v)^{l(y)-l(x)} v^{-l\left(y_{\mathbf{i}}\right)} \bar{P}_{y x}\left|\lambda \cdot x^{-1} y\right\rangle,
$$

where the sum is over all the $y$ such that $y \leq x$ and $\lambda \cdot x^{-1} y \in \Pi_{l}$.

(b) For all $\lambda \in \Pi_{l}$ the coordinates of $\mathbf{b}_{\lambda}^{+}$in the wedges are some parabolic KazhdanLusztig polynomials (w.r.t. the parabolic subgroup $\mathfrak{S}_{l} \subset \widehat{\mathfrak{S}}_{l}$ ).

Now, suppose that $l \leq n$. Let consider $\mathbf{i}, \mathbf{j} \in A_{l}^{n}, \mathbf{n} \in M^{+}$and $\mathbf{m}=\mathbf{n}^{\mathbf{j}} \in M_{\mathbf{j i}} \cap M^{+}$. If $x \in \mathfrak{S}^{\mathbf{i}}$ is such that $(\mathbf{i}) x \in P_{l}^{++}$then Section 7 gives

$$
\mathbf{f}_{O_{\mathbf{n}}}\left(\wedge \mathbf{x}_{(\mathbf{i}) x}\right)=v^{l\left(\omega_{\mathbf{i}}\right)+y(\mathbf{m})} T_{\mathbf{m}} \tilde{T}_{x} e^{-} \in e_{\mathbf{j}} \widehat{\mathbf{H}}_{l} e^{-} .
$$

In particular if $\mathbf{i}=(\rho) \omega \in A_{l}^{n}$ and $x=\omega$ we get

$$
\mathbf{f}_{O_{\mathbf{n}}}\left(\wedge \mathbf{x}_{\emptyset}\right)=v^{l\left(\omega_{\mathbf{j}}\right)} e_{\mathbf{j}} \tilde{T}_{m} \tilde{T}_{\omega} e^{-},
$$

where $m \in \mathbf{m}$ is the smallest element. Let suppose that $\mathbf{f}_{O_{\mathbf{n}}}\left(\wedge \mathbf{x}_{\emptyset}\right) \neq 0$. Let $\mathfrak{S}^{l}$ be the set of minimal length representatives of the cosets in $\widehat{\mathfrak{S}}_{l} / \mathfrak{S}_{l}$. Then Lemma 9.3 implies that $m=y t$ with $t \in \mathfrak{S}_{l}$ and $y \in \mathfrak{S}^{\mathbf{j}} \cap \mathfrak{S}^{l}$ such that $S_{\mathbf{j}} y \cap y S_{l}=\emptyset$. Then,

$$
\mathbf{f}_{O_{\mathbf{n}}}\left(\wedge \mathrm{x}_{\emptyset}\right)=v^{l\left(\omega_{\mathbf{j}}\right)} e_{\mathbf{j}} \tilde{T}_{y} \tilde{T}_{t} \tilde{T}_{\omega} e^{-}=v^{l\left(\omega_{\mathbf{j}}\right)} e_{\mathbf{j}} \tilde{T}_{y} \tilde{T}_{\omega} \tilde{T}_{\omega t \omega} e^{-}=(-v)^{l(t)} \wedge \mathrm{x}_{(\mathbf{j}) y \omega} \in \bigoplus_{\lambda} \mathbb{S}|\lambda\rangle .
$$

As a consequence if $l \leq n$ then $\mathbf{B}_{l}^{+}=\mathbf{B}_{l}$.

Conjecture. The bases $\mathbf{B}_{l}$ and $\mathbf{B}_{l}^{+}$coincide for all $l$.

\section{Proof of Theorem 6.3.}


10.1. Let $\otimes^{\infty}$ be the free $\mathbb{A}$-module linearly generated by the semi-infinite monomials

$$
\otimes \mathrm{x}_{\mathbf{i}}=\mathrm{x}_{i_{1}} \otimes \mathrm{x}_{i_{2}} \otimes \mathrm{x}_{i_{3}} \otimes \cdots
$$

where $\mathbf{i}=\left(i_{1}, i_{2}, \ldots\right)$ is a sequence of integers such that $i_{k}=1-k$ for $k>>1$. The affine Hecke algebra of type $\mathfrak{g l}_{\infty}$ acts on $\bigotimes^{\infty}$ via formulas (8.b) and (8.c). Set $\Omega^{\infty}=\sum_{i} \operatorname{Im}\left(T_{i}+1\right) \subset \bigotimes^{\infty}$. As above $\wedge \mathrm{x}_{\mathbf{i}}$ is the class of $\otimes \mathrm{x}_{\mathbf{i}}$ in $\otimes^{\infty} / \Omega^{\infty}$. The formulas in Section 8 and [KMS, Lemma 2.2] imply that for all $\alpha \in \mathbb{N} \mathbb{Z} / n \mathbb{Z}$ we have

(a) $\quad \forall \mathbf{i} \quad \exists l \in \mathbb{N}^{\times} \quad$ such that $\mathbf{f}_{\alpha}\left(\wedge \mathrm{x}_{\mathbf{i}}\right)=\mathbf{f}_{\alpha}\left(\mathrm{x}_{i_{1}} \wedge \cdots \wedge \mathrm{x}_{i_{l}}\right) \wedge \mathrm{x}_{i_{l+1}} \wedge \mathrm{x}_{i_{l+2}} \wedge \ldots$

Thus the action of $\mathbf{U}_{n}^{-}$on $\bigwedge^{l}$ induces an action on $\bigwedge^{\infty}$.

Lemma. The map $|\lambda\rangle \mapsto \wedge \mathrm{x}_{\mathbf{i}}$, where $i_{k}=1+\lambda_{k}-k$, gives an embedding of the representation of $\mathbf{U}_{n}^{-}$on $\bigwedge^{\infty}$ given in Section 6 into $\otimes^{\infty} / \Omega^{\infty}$.

Proof. The proof goes by a direct computation. First observe that $\wedge \mathrm{x}_{\mathbf{i}}$ and $|\lambda\rangle$ have the same weight for any $\lambda \in \Pi$ if $\mathbf{i}$ is the sequence such that $i_{k}=1+\lambda_{k}-k$. Fix $\lambda \in \Pi$ and $\alpha \in \mathbb{N}^{\mathbb{Z} / n \mathbb{Z}}$. Fix $\mathbf{i}$ as above. Formula 6.2.a gives

$$
\mathbf{f}_{\alpha}\left(\wedge \mathbf{x}_{\mathbf{i}}\right)=\mathbf{f}_{\alpha}(|\lambda\rangle)=\sum_{\text {as.t. } \bar{a}=\alpha} \gamma_{a}\left(\mathbf{f}_{\alpha}\right) \prod_{\substack{j<i \\ \bar{\imath}=\bar{\jmath}}} \mathbf{k}_{i}^{a_{j}}(|\lambda\rangle) .
$$

Moreover Remark 6.1 implies that $\gamma_{a}\left(\mathbf{f}_{\alpha}\right)$ is $v^{h(a)}$ times the product of the $\mathbf{f}_{i}^{\left(a_{i}\right)}$ 's ordered from $i=-\infty$ to $\infty$. Using the formulas in Section 4 we first observe that $\mathbf{f}_{i}^{(2)}$ acts by zero on the Fock space for any $i$. The elements $|\lambda\rangle$ and $\otimes \mathbf{x}_{\mathbf{i}}$ have the same weight with respect to $\mathbf{k}_{i}$. Thus we get

$$
\mathbf{f}_{\alpha}\left(\wedge \mathbf{x}_{\mathbf{i}}\right)=\sum_{\mathbf{n}} v^{e(\mathbf{i}, \mathbf{i}+\mathbf{n})} \wedge \mathbf{x}_{\mathbf{i}+\mathbf{n}}
$$

where $\mathbf{n}=\left(n_{1}, n_{2}, \ldots\right) \in\{0,1\}^{\mathbb{N}^{\times}}$describes the set of all sequences such that $\alpha=$ $\sum_{s \geq 1} n_{s} \epsilon_{\bar{\imath}_{s}}$ and

$$
\begin{gathered}
e(\mathbf{i}, \mathbf{i}+\mathbf{n})=\sum_{i_{k}>i_{l}} n_{l} \delta_{\bar{\imath}_{l}, \bar{\imath}_{k}}-\sum_{i_{k}>1+i_{l}} n_{l} \delta_{\bar{\imath}_{l}+1, \bar{\imath}_{k}}- \\
-\sum_{i_{k}<i_{l}} n_{l} n_{k} \delta_{\bar{\imath}_{l}, \bar{\nu}_{k}}+\sum_{1+i_{k}<i_{l}} n_{l} n_{k} \delta_{\bar{l}_{l}, \bar{\imath}_{k}+1} .
\end{gathered}
$$

If $\wedge \mathbf{x}_{\mathbf{i}+\mathbf{n}} \neq 0$ then $e(\mathbf{i}, \mathbf{i}+\mathbf{n})=\sum_{i_{k}>i_{l}} n_{l}\left(1-n_{k}\right)\left(\delta_{\bar{l}_{l}, \bar{\imath}_{k}}-\delta_{\bar{\imath}_{l}+1, \bar{\nu}_{k}}\right)$. On the other hand the formula in Section 8.3 gives

$$
\mathbf{f}_{\alpha}\left(\wedge \mathbf{x}_{\mathbf{i}}\right)=\sum_{\mathbf{n}} v^{c(\mathbf{i}, \mathbf{i}+\mathbf{n})} \wedge \mathbf{x}_{\mathbf{i}+\mathbf{n}}
$$

where $\mathbf{n}$ describes the same set and

$$
c(\mathbf{i}, \mathbf{i}+\mathbf{n})=-\sum_{1 \leq k<l} n_{l}\left(1-n_{k}\right) n\left(\epsilon_{\bar{\imath}_{l}}, \epsilon_{\bar{\imath}_{k}}\right) .
$$


We are through (recall that $\mathbf{i}$ is decreasing).

Theorem 6.3 follows from Proposition 9.2 and Lemma 10.1.

10.2. The involution $\psi$ on $\bigwedge^{l}$ induces the semilinear involution $\psi$ on $\bigwedge^{\infty}$ such that,

$$
\forall \mathbf{i}, \quad l \geq \sum_{k}\left(i_{k}-1+k\right) \Rightarrow \psi\left(\wedge \mathrm{x}_{\mathbf{i}}\right)=\psi\left(\mathrm{x}_{i_{1}} \wedge \cdots \wedge \mathrm{x}_{i_{l}}\right) \wedge \mathrm{x}_{i_{l+1}} \wedge \mathrm{x}_{i_{l+2}} \wedge \ldots
$$

Proposition 9.3 implies that $\psi$ coincides with the involution on $\bigwedge^{\infty}$ used in [LT]. In [LT] Leclerc and Thibon have defined two bases $\mathbf{B}^{ \pm}=\left\{\mathbf{b}_{\lambda}^{ \pm} \mid \lambda \in \Pi\right\}$ in $\wedge^{\infty}$ such that for all $\lambda$

$$
\psi\left(\mathbf{b}_{\lambda}^{ \pm}\right)=\mathbf{b}_{\lambda}^{ \pm}, \quad \mathbf{b}_{\lambda}^{-}-|\lambda\rangle \in \bigoplus_{\mu<\lambda} v^{-1} \overline{\mathbb{S}}|\mu\rangle \quad \text { and } \quad \mathbf{b}_{\lambda}^{+}-|\lambda\rangle \in \bigoplus_{\mu<\lambda} v \mathbb{S}|\mu\rangle .
$$

Thus, Conjecture 9.5 is equivalent to

Conjecture. The bases $\mathbf{B}$ and $\mathbf{B}^{+}$coincide.

Set $\mathbf{b}_{\lambda}=\sum_{\mu} d_{\mu \lambda}|\mu\rangle$ and $\mathbf{b}_{\lambda}^{ \pm}=\sum_{\mu} e_{\mu \lambda}^{ \pm}|\mu\rangle$. Conjecture 10.2 is precisely $d_{\lambda \mu}=e_{\lambda \mu}^{+}$.

\section{Proof of the Decomposition Conjecture.}

Let $\varepsilon$ be a $n$-th root of unity. The quantized Schur algebra $\mathbf{S}_{n, l}$ is the subalgebra of $\widehat{\mathbf{S}}_{n, l}$ spanned by the elements $T_{\mathbf{m}}$ with $\mathbf{m} \in \mathfrak{S}_{\mathbf{i}} \backslash \mathfrak{S}_{l} / \mathfrak{S}_{\mathbf{j}}$ (see Subsection 7.4). Fix $l \leq k$. Let consider the subalgebra

$$
\mathbf{S}_{l}=\mathbf{S}_{k, l} \cap \bigoplus_{\lambda, \mu \in \Pi(l)} \widehat{\mathbf{H}}_{\mathbf{i}_{\lambda} \mathbf{i}_{\mu}}
$$

where $\mathbf{i}_{\lambda}=(1-k)^{\lambda_{1}}(2-k)^{\lambda_{2}} \ldots 0^{\lambda_{k}}$ for any $\lambda=\left(\lambda_{1} \geq \lambda_{2} \geq \ldots\right) \in \Pi(l)$. We want to compute the decomposition matrices of the simple $\mathbf{S}_{l}$-modules under the specialization $v \mapsto \varepsilon$. The algebra $\mathbf{S}_{k, l}$ is Morita equivalent to $\mathbf{S}_{l}$. For any $t \in$ $\mathbb{C}^{\times}$let $\mathbf{S}_{l \mid t}$ be the specialization of $\mathbf{S}_{l}$ at $v=t$. The simple modules of $\mathbf{S}_{l \mid t}$ are parametrized by $\Pi(l)$. For any $k$ let $\mathbf{U}\left(\mathfrak{g l}_{k}\right)$ be the Lusztig integral form of the quantized enveloping algebra of $\mathfrak{g l}_{k}$ and let $\mathbf{U}_{\varepsilon}\left(\mathfrak{g l}_{k}\right)$ be the specialization at $v=\varepsilon$. The set $\Pi_{k}$ is identified with the set of dominant weights of $\mathfrak{g l}_{k}$ with non-negative components. If $\lambda \in \Pi_{k}$, let $V_{\lambda}$ and $W_{\lambda}$ be respectively the simple and the Weyl $\mathbf{U}_{\varepsilon}\left(\mathfrak{g l}_{k}\right)$-module with highest weight $\lambda$. There exists a surjective map $\pi: \mathbf{U}_{\varepsilon}\left(\mathfrak{g l}_{k}\right) \rightarrow$ $\mathbf{S}_{k, l \mid \varepsilon}$ (see [D2]). If $\lambda \in \Pi(l)$ let $L_{\lambda}, M_{\lambda}$, be the simple and the Specht $\mathbf{S}_{k, l \mid \varepsilon}$-modules such that

$$
\pi^{*}\left[L_{\lambda}\right]=\left[V_{\lambda^{\prime}}\right] \text { and } \pi^{*}\left[M_{\lambda}\right]=\left[W_{\lambda^{\prime}}\right]
$$

in the Grothendieck ring.

Theorem. The specialization at $v=1$ of the matrix $\left(e_{\lambda \mu}^{+}\right)_{\lambda \mu}, \lambda, \mu \in \Pi(l)$, is the decomposition matrix of the Specht modules of $\mathbf{S}_{l}$.

Proof. The Lusztig conjecture (proved by Kashiwara-Tanisaki and Kazhdan-Lusztig) gives the multiplicity of $W_{\mu}$ in $V_{\lambda}$. More precisely we have

$$
\left[V_{\lambda}: W_{\mu}\right]=\sum_{y}(-1)^{l(y x)} P_{y x}(1)
$$


where $x \in \widehat{\mathfrak{S}}_{l}$ is minimal such that $\nu=\lambda \cdot x^{-1}$ satisfies

$$
\nu_{i}<\nu_{i+1} \quad \forall i=1,2, \ldots, k-1, \quad \nu_{1}-\nu_{k} \geq 1-k-n,
$$

and $\mu=\lambda \cdot x^{-1} y$. According to Theorem 9.5.a, the Lusztig Conjecture is equivalent to

$$
\left[L_{\lambda^{\prime}}\right]=\sum_{\mu} e_{\lambda \mu}^{-}(1)\left[M_{\mu^{\prime}}\right], \quad \forall \lambda \in \Pi_{k}
$$

Recall that $\left(e_{\lambda \mu}^{+}\right)_{\lambda \mu}=\left(\bar{e}_{\lambda^{\prime} \mu^{\prime}}^{-}\right)_{\lambda \mu}^{-1}$ (see [LT, Section 4]). Thus

$$
(a) \Longleftrightarrow\left[M_{\lambda}\right]=\sum_{\mu} e_{\lambda \mu}^{+}(1)\left[L_{\mu}\right]
$$

\section{The Lusztig conjecture.}

12.1. Let $F$ be the variety of partial flags in $\mathbb{C}^{l}$ of the type

$$
\{0\} \subseteq F_{1} \subseteq F_{2} \subseteq \cdots \subseteq F_{k}=\mathbb{C}^{l} .
$$

The linear group $G L_{l}$ acts diagonaly on $F \times F$. Let $Z \subset T^{*} F \times T^{*} F$ be the Steinberg variety $(Z$ is a reducible variety whose irreducible components are the closure of the conormal bundles to the $G L_{l}$-orbits in $\left.F \times F\right)$. The group $G=$ $G L_{l} \times \mathbb{C}^{\times}$acts naturally on $Z$ : the linear group acts diagonaly and $z \in \mathbb{C}^{\times}$ acts by multiplication by $z^{-2}$ along the fibers. The complexified Grothendieck group of equivariant coherent sheaves on $Z$, denoted by $\mathbf{K}_{k, l}$, is endowed with an associative convolution product (see $[\mathrm{GV}]$, [V2]) denoted by $\star$. For any $z \in \mathbb{C}^{\times}$, a parametrization of the simple modules of the specialized algebra $\mathbf{K}_{k, l \mid v=z}$ is given in [GV] (see in [V2] the remark after Theorem 4 for the case of roots of unity): the simple modules are labelled by orbits of pairs $(s, x) \in \mathrm{GL}_{l} \times \mathfrak{g l}_{l}$ where $s$ is semi-simple, $x^{k}=0$, and $s x s^{-1}=z^{-2} x$. As usual the $G L_{l}$-orbit of $x$ is labelled by the partition $\lambda \in \Pi(l)$ such that $\lambda_{i}$ is the length of the $i$-th Jordan block of $x$. Then $\lambda^{\prime} \in \Pi(l) \cap \Pi_{k}$. The orbits of the pairs $(s, x)$ such that the spectrum of $s$ is in $z^{2 \mathbb{Z}}$ are labelled by isomorphism class of nilpotent representations of $\Gamma_{\infty}$ if $z$ is generic and of $\Gamma_{n}$ if $z=\varepsilon$ (recall that $\varepsilon^{2}$ is a primitive $n$-th root of unity). Let $\Omega_{k, l}$ and $\Omega_{k, l}^{\infty}$ be the corresponding sets of isomorphism classes of representations of $\Gamma_{n}$ and $\Gamma_{\infty}$. If $O \in \Omega_{k, l}^{\infty}$ (resp. $\left.O \in \Omega_{k, l}\right)$ let $L_{O}^{\infty}\left(\right.$ resp. $\left.L_{O}\right)$ be the simple $\mathbf{K}_{k, l}$-module labelled by $O$. Similarly let $M_{O}^{\infty}$ and $M_{O}$ be the standard modules labelled by $O$ (see [V2]). Let $[M]$ be the class of the module $M$ in the complexified Grothendieck ring. Let $\widehat{\mathbf{R}}_{n}$ and $\widehat{\mathbf{R}}_{\infty}$ be the linear span of the elements $\left[L_{O}\right]$ and $\left[L_{O}^{\infty}\right]$ where $O \in \Omega_{k, k}$ or $O \in \Omega_{k, k}^{\infty}$ and $k \geq 1$. The restricted dual $\widehat{\mathbf{R}}_{n}^{*}$ (resp. $\widehat{\mathbf{R}}_{\infty}^{*}$ ) is spanned by the linear forms $l_{O}\left(\right.$ resp. $\left.l_{O}^{\infty}\right)$ such that

$$
l_{O}\left(\left[L_{O^{\prime}}\right]\right)=\delta_{O, O^{\prime}} \quad \text { and } \quad l_{O}^{\infty}\left(\left[L_{O^{\prime}}^{\infty}\right]\right)=\delta_{O, O^{\prime}} .
$$

12.2. The quantized enveloping algebra of $\widehat{\mathfrak{g l}}_{k}$ is generated by elements $\mathbf{e}_{i, s}, \mathbf{f}_{i, s}$, $\mathbf{h}_{j, t}$ and $\mathbf{k}_{j}^{ \pm 1}\left(0<i<k, 0<j \leq k, s \in \mathbb{Z}, t \in \mathbb{Z}^{\times}\right)$which satisfy the relations 
of the Drinfeld new presentation. Let $\mathbf{U}\left(\widehat{\mathfrak{g l}}_{k}\right)$ be the $\mathbb{A}$-subalgebra generated by the elements $\mathbf{e}_{i, s}^{(m)}, \mathbf{f}_{i, s}^{(m)},[t]^{-1} \mathbf{h}_{j, t}$ and $\mathbf{k}_{j}^{ \pm 1}$. For any $z \in \mathbb{C}^{\times}$let $\mathbf{U}_{z}\left(\widehat{\mathfrak{g l}}_{k}\right)$ be its specialization at $v=z$. In [GV], [V2], is defined a surjective algebra homomorphism $\Psi_{k, l}: \mathbf{U}\left(\widehat{\mathfrak{g l}}_{k}\right) \otimes_{\mathbb{A}} \mathbb{C}(v) \rightarrow \mathbf{K}_{k, l} \otimes_{\mathbb{A}} \mathbb{C}(v)$. It is proved in $[\mathrm{S}]$ that $\Psi_{k, l}$ restricts to a surjective homomorphism $\mathbf{U}\left(\widehat{\mathfrak{g l}}_{k}\right) \rightarrow \mathbf{K}_{k, l}$. Observe that the restriction of a simple $\mathbf{U}_{z}\left(\widehat{\mathfrak{g l}}_{k}\right)$-module to $\mathbf{U}_{z}\left(\widehat{\mathfrak{s l}}_{k}\right)$ is simple. Thus $\Psi_{k, l}^{*} L_{O}$ for $O \in \Omega_{k, l}$ (resp. $\Psi_{k, l}^{*} L_{O}^{\infty}$ for $\left.O \in \Omega_{k, l}^{\infty}\right)$, may be viewed as a simple $\mathbf{U}_{z}\left(\widehat{\mathfrak{s l}}_{k}\right)$-module when $z=\varepsilon$ (resp. $z$ generic). Recall that there is an algebra homomorphism ev $: \mathbf{U}\left(\widehat{\mathfrak{s l}}_{k}\right) \rightarrow \mathbf{U}\left(\mathfrak{g l}_{k}\right)$ such that

$$
\begin{aligned}
& e v\left(\mathbf{e}_{0}\right)=v^{-1}\left\{\mathbf{f}_{k-1},\left\{\mathbf{f}_{k-2}, \ldots\left\{\mathbf{f}_{2}, \mathbf{f}_{1}\right\} \ldots\right\}\right\} \mathbf{k}_{k} \mathbf{k}_{k-1} \\
& e v\left(\mathbf{f}_{0}\right)=(-1)^{k} v^{k-1}\left\{\mathbf{e}_{k-1},\left\{\mathbf{e}_{k-2}, \ldots\left\{\mathbf{e}_{2}, \mathbf{e}_{1}\right\} \ldots\right\}\right\} \mathbf{k}_{k}^{-1} \mathbf{k}_{k-1}^{-1} \\
& e v\left(\mathbf{f}_{i}\right)=\mathbf{f}_{i}, \quad \operatorname{ev}\left(\mathbf{e}_{i}\right)=\mathbf{e}_{i}, \quad i=1,2, \ldots, k-1,
\end{aligned}
$$

where $\{x, y\}=x y-v^{-1} y x$. If $\lambda \in \Pi_{k}$ let $V_{\lambda}$ (resp. $\left.V_{\lambda}^{\infty}\right)$ be the simple $\mathbf{U}_{z}\left(\mathfrak{g l}_{k}\right)$ modules with highest weight $\lambda$ where $z=\varepsilon$ (resp. $z$ generic). The Drinfeld polynomials of $L_{O}$ and $L_{O}^{\infty}$ are computed in [V2]. If $\lambda \in \Pi(k)$, then $\Psi_{k, k}^{*} L_{O_{\lambda}}$ and $\Psi_{k, k}^{*} L_{O_{\lambda}}^{\infty}$ are the pull-backs of the modules $V_{\lambda^{\prime}}$ and $V_{\lambda^{\prime}}^{\infty}$ by the evaluation map ev (see [CP, Proposition 12.2.13]). Let $\mathbf{R}_{n}$ and $\mathbf{R}_{\infty}$ be the linear span of the classes $\left[V_{\lambda}\right]$ and $\left[V_{\lambda}^{\infty}\right]$ for all $\lambda$ and all $k$. The restricted dual spaces $\mathbf{R}_{n}^{*}$ and $\mathbf{R}_{\infty}^{*}$ are spanned by the linear forms $l_{\lambda}$ and $l_{\lambda}^{\infty}$ such that

$$
l_{\lambda}\left(\left[V_{\mu^{\prime}}\right]\right)=\delta_{\lambda \mu} \quad \text { and } \quad l_{\lambda}^{\infty}\left(\left[V_{\mu^{\prime}}^{\infty}\right]\right)=\delta_{\lambda \mu} .
$$

The element $\left[V_{\lambda}^{\infty}\right]$ may be viewed as the class in $\mathbf{R}_{n}$ of the Weyl module $W_{\lambda}$ with highest weight $\lambda$. Let $s_{\lambda} \in \mathbf{R}_{n}^{*}$ be such that

$$
s_{\lambda}\left(\left[W_{\mu^{\prime}}\right]\right)=\delta_{\lambda \mu}
$$

12.3. In this subsection $\mathbf{U}_{n}^{-}, \mathbf{U}_{\infty}^{-}$and $\bigwedge^{\infty}$ stand for their specializations at $v=1$.

Theorem. The linear isomorphism $\mathbf{R}_{n}^{*} \stackrel{\sim}{\rightarrow} \bigwedge^{\infty}$ such that $s_{\lambda} \mapsto|\lambda\rangle$ maps $l_{\lambda}$ to $\mathbf{b}_{\lambda}$.

Proof. First observe that the classes of the standard modules $\left[M_{O}\right]$ and $\left[M_{O}^{\infty}\right]$ form a basis of the spaces $\widehat{\mathbf{R}}_{n}$ and $\widehat{\mathbf{R}}_{\infty}$. Let $m_{O}$ and $m_{O}^{\infty}$ be the elements of the dual basis. To avoid confusions let $\mathbf{f}_{O}^{\infty}, \mathbf{b}_{O}^{\infty}$, denote the generatrors of $\mathbf{U}_{\infty}^{-}$. The multiplicity formula $[\mathrm{GV}$, Theorem 6.6] implies that there are two linear isomorphisms

$$
\iota_{n}: \mathbf{U}_{n}^{-} \rightarrow \widehat{\mathbf{R}}_{n}^{*} \quad \text { and } \quad \iota_{\infty}: \mathbf{U}_{\infty}^{-} \rightarrow \widehat{\mathbf{R}}_{\infty}^{*}
$$

such that

$$
\iota_{n}\left(\mathbf{f}_{O}\right)=m_{O}, \quad \iota_{n}\left(\mathbf{b}_{O}\right)=l_{O}, \quad \iota_{\infty}\left(\mathbf{f}_{O}^{\infty}\right)=m_{O}^{\infty}, \quad \iota_{\infty}\left(\mathbf{f}_{O}^{\infty}\right)=l_{O}^{\infty} .
$$

The spaces $\mathbf{R}_{n}^{*}$ and $\mathbf{R}_{\infty}^{*}$ are identified with $\bigwedge^{\infty}$ via the maps

$$
s_{\lambda} \mapsto|\lambda\rangle \quad \text { and } \quad l_{\lambda}^{\infty} \mapsto|\lambda\rangle .
$$


We obtain the following commutative square

$$
\begin{aligned}
& \bigwedge^{\infty}=\mathbf{R}_{n}^{*} \stackrel{\sim}{\rightarrow} \mathbf{R}_{\infty}^{*}=\bigwedge^{\infty} \\
& \mathbf{U}_{n}^{-}=\widehat{\mathbf{R}}_{n}^{*} \rightarrow \widehat{\mathbf{R}}_{\infty}^{\uparrow}=\mathbf{U}_{\infty}^{-},
\end{aligned}
$$

where the horizontal arrows are the dual of the specialization maps and the vertical arrows are the dual of the evaluation maps. By definition the upper arrow maps $s_{\lambda}$ to $l_{\lambda}^{\infty}$ and both elements are identified with the vacuum vector $|\lambda\rangle$. The right vertical arrow is such that

$$
\mathbf{b}_{O}^{\infty}=l_{O}^{\infty} \mapsto l_{\lambda}^{\infty}=|\lambda\rangle \quad \text { if } \quad O=O_{\lambda}, \quad \mathbf{b}_{O}^{\infty} \mapsto 0 \quad \text { else. }
$$

By Proposition 5 it is the quotient map

$$
\mathbf{U}_{\infty}^{-} \rightarrow \bigwedge^{\infty}, \quad u \mapsto u(|\emptyset\rangle)
$$

Suppose first that the lower horizontal arrow is the map $\gamma$ introduced in Section 6 . Then the left vertical arrow is the quotient map

$$
\mathbf{U}_{n}^{-} \rightarrow \bigwedge^{\infty}, \quad u \mapsto u(|\emptyset\rangle)
$$

Hence $(a)$ implies that the left vertical arrow maps $l_{O_{\lambda}}$ to $\mathbf{b}_{\lambda}$. Since this arrow is the transpose of the evaluation map we get $l_{\lambda}=\mathbf{b}_{\lambda}$ and we are through. By Subsection 6.4, to prove that the map $\widehat{\mathbf{R}}_{n}^{*} \rightarrow \widehat{\mathbf{R}}_{\infty}^{*}$ is $\gamma$ we are reduced to prove that if $r\left(O^{\prime}\right) \subseteq O$ then $\left[M_{O^{\prime}}^{\infty}\right]$ specializes to $\left[M_{O}\right]$. This is obvious by the localization theorem in equivariant $K$-theory.

12.4. Theorem 12.3 implies that $\left[V_{\lambda}^{\infty}\right]=\sum_{\mu} d_{\lambda^{\prime} \mu^{\prime}}(1)\left[V_{\mu}\right]$. According to Section 11 the Lusztig Conjecture can be written as

$$
\left[W_{\lambda}\right]=\sum_{\mu} e_{\lambda^{\prime} \mu^{\prime}}^{+}(1)\left[V_{\mu}\right] \quad \forall \lambda,
$$

which is precisely Conjecture 10.2 .

\section{Proof of Proposition 6.1.}

13.1. Fix $\Gamma=\Gamma_{n}$ or $\Gamma_{\infty}$. Let $\mathcal{S}_{d}$ be the set of finite sequences $\mathbf{d}=\left(d^{1}, d^{2}, \ldots, d^{l}\right)$ of elements in $\mathbb{N}^{(I)}$ such that $\sum_{k} d^{k}=d$. Fix a $I$-graded vector space $V$ of dimension $d$. For each $\mathbf{d} \in \mathcal{S}_{d}$ let $F_{\mathbf{d}}$ be the set of flags of $V$ of type d, i.e. $F_{\mathbf{d}}$ is the set of filtrations $F=\left(\{0\}=F^{0} \subseteq F^{1} \subseteq \cdots \subseteq F^{l}=V\right)$ such that $F^{k}$ is $I$-graded and has dimension $d^{1}+d^{2}+\cdots+d^{k}$. Given $x \in E_{V}$ we say that a flag $F \in F_{\mathbf{d}}$ is $x$-stable if $x\left(F^{k}\right) \subseteq F^{k-1}$ for all $k$. Let $\tilde{F}_{\mathbf{d}}$ be the variety of all pairs $(x, F)$ such that $x \in E_{V}$ and $F \in F_{\mathbf{d}}$ is $x$-stable. The group $G_{V}$ acts on $\tilde{F}_{\mathbf{d}}$ in the obvious way. Let $\pi_{\mathbf{d}}: \tilde{F}_{\mathbf{d}} \rightarrow E_{V}$ be the first projection. The map $\pi_{\mathbf{d}}$ commutes to $G_{V}$. Thus

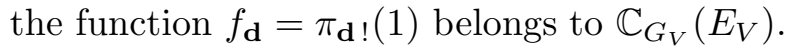

Lemma. (a) The space $\mathbb{C}_{G_{V}}\left(E_{V}\right)$ is linearly spanned by the elements $f_{\mathbf{d}}$ with $\mathbf{d} \in \mathcal{S}_{d}$. 
(b) For any $a, b \in \mathbb{N}^{(I)}$ and any $\mathbf{a} \in \mathcal{S}_{a}, \mathbf{b} \in \mathcal{S}_{b}$, we have $f_{\mathbf{a}} \circ f_{\mathbf{b}}=q^{-m(b, a)} f_{\mathbf{a b}}$ where $\mathbf{a b} \in \mathcal{S}_{a+b}$ is the sequence $\mathbf{a}$ followed by the sequence $\mathbf{b}$.

Proof. Claim $(b)$ is proved as in [L2, Lemma 3.2.b]. Let us prove claim $(a)$. If a flag $F$ is $x$-stable then $F^{k} \subseteq \operatorname{Ker}\left(x^{k}\right)$. Thus if $\mathbf{d} \in \mathcal{S}_{d}$ is such that

$$
d^{1}+d^{2}+\cdots+d^{k}=\operatorname{dim} \operatorname{Ker}\left(x^{k}\right) \quad \forall k=1,2,3, \ldots,
$$

then $\pi_{\mathbf{d}}^{-1}(x)$ is reduced to the single flag

$$
\{0\} \subseteq \operatorname{Ker}(x) \subseteq \operatorname{Ker}\left(x^{2}\right) \subseteq \cdots \subseteq V
$$

In particular $f_{\mathbf{d}}(x)=1$. Moreover, in this case $f_{\mathbf{d}}$ is supported on the $G_{V}$-orbits of the $y$ 's such that

$$
\operatorname{dim} \operatorname{Ker}\left(x^{k}\right) \leq \operatorname{dim} \operatorname{Ker}\left(y^{k}\right) \quad \forall k=1,2,3, \ldots,
$$

i.e. $y \in \overline{G_{V} \cdot x}$. We are through.

Remark. It is easy to see that for any $d \in \mathbb{N} \mathbb{Z} / n \mathbb{Z}$ and $\mathbf{d}=(d)$ we have $f_{\mathbf{d}}=\mathbf{f}_{d}$. Thus Proposition 3.5 is a consequence of $(a)$ and $(b)$.

13.2. We fix a $\mathbb{Z}$-graded vector space $V$ of dimension $d$. Let $\bar{V}$ be the associated $\mathbb{Z} / n \mathbb{Z}$-graded vector space, of dimension $\bar{d}$. The space $\bar{V}$ is endowed with the $\mathbb{Z}$ filtration whose associated graded is identified with $V$. Fix $\overline{\mathbf{d}} \in \mathcal{S}_{\bar{d}}$. We have the following commutative diagram

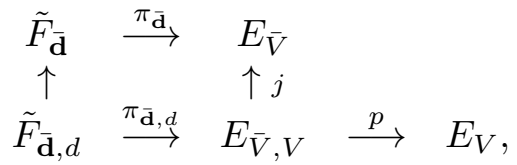

where $\tilde{F}_{\overline{\mathbf{d}}, d}=\pi_{\overline{\mathbf{d}}}^{-1}\left(E_{\bar{V}, V}\right)$ and the vertical arrows are the embeddings. We have clearly

$$
p_{!} j^{*}\left(f_{\overline{\mathbf{d}}}\right)=\left(p \pi_{\overline{\mathbf{d}}, d}\right) !(1) .
$$

Let $\mathcal{S}_{\overline{\mathbf{d}}, d} \subset \mathcal{S}_{d}$ be the set of sequences $\mathbf{d}$ such that $\sum_{i \in \bar{\imath}} d_{i}^{k}=\bar{d}_{\bar{\imath}}^{k}$ for any $k$ and $\bar{\imath}$. If $\mathbf{d} \in \mathcal{S}_{\overline{\mathbf{d}}, d}$ let $\tilde{F}_{\overline{\mathbf{d}}, \mathbf{d}} \subset \tilde{F}_{\overline{\mathbf{d}}, d}$ be the set of pairs $(x, F)$ such that the associated graded of $F^{k}$ with respect to the filtration induced by the $\mathbb{Z}$-filtration on $\bar{V}$ has dimension $d^{1}+d^{2}+\cdots+d^{k}$. The sets $\tilde{F}_{\overline{\mathbf{d}}, \mathbf{d}}$ form a partition of $\tilde{F}_{\overline{\mathbf{d}}, d}$. We have a commutative square

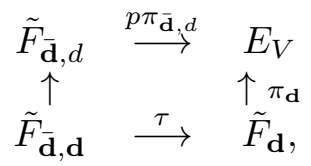

where the left vertical arrow is the inclusion and $\tau$ maps the pair $(x, F)$ to the associated graded. Thus

$$
\left(p \pi_{\overline{\mathbf{d}}, d}\right) !(1)=\sum_{\mathbf{d} \in \mathcal{S}_{\overline{\mathbf{d}}, d}}\left(\pi_{\mathbf{d}} \tau\right) !(1) .
$$


Lemma. The map $\tau$ is a vector bundle of rank

$$
r(\mathbf{d})=\sum_{k \geq l} \sum_{\substack{i>j \\ \bar{\imath}=\bar{\jmath}}} d_{j}^{k} d_{i+1}^{l}+\sum_{k<l} \sum_{\substack{i>j \\ \bar{\imath}=\bar{\jmath}}} d_{j}^{k} d_{i}^{l} .
$$

Proof. The proof goes as the proof of [L2, Lemma 4.4]. More precisely fix $(x, F) \in$ $\tilde{F}_{\mathbf{d}}$ and compute the fiber $\tau^{-1}(x, F)$. Giving a $\mathbb{Z} / n \mathbb{Z}$-graded subspace $\bar{F}^{k} \in \bar{V}$ of dimension $\bar{d}^{1}+\bar{d}^{2}+\cdots+\bar{d}^{k}$ whose associated $\mathbb{Z}$-graded is $F^{k}$ is the same as giving a map

$$
z^{k}=\oplus z_{i j}^{k} \in \bigoplus_{\substack{i>j \\ \bar{\imath}=\bar{\jmath}}} \operatorname{Hom}\left(F_{j}^{k}, V_{i} / F_{i}^{k}\right) .
$$

Then $\bar{F}^{k} \subset \bar{F}^{k+1}$ if and only if $z^{k+1}=z^{k}: F^{k} \rightarrow V / F^{k+1}$. On the other hand giving $\bar{x} \in E_{\bar{V}, V}$ such that $p(\bar{x})=x$ is the same as giving a map

$$
y=\oplus y_{i+1, j} \in \bigoplus_{\substack{i>j \\ \bar{\imath}=\bar{\jmath}}} \operatorname{Hom}\left(V_{j}, V_{i+1}\right) .
$$

Then $\bar{F}$ is $\bar{x}$-stable if and only if

$$
z_{i+1, j+1}^{k} \circ x_{j}-x_{i} \circ z_{i j}^{k}-y_{i+1, j}=0: F_{j}^{k} \rightarrow V_{i+1} / F_{i+1}^{k} \text {. }
$$

The Lemma results from a direct computation.

The Lemma and $(c),(d)$, give

$$
\gamma_{d}\left(f_{\overline{\mathbf{d}}}\right)=\sum_{\mathbf{d} \in \mathcal{S}_{\overline{\mathbf{d}}, d}} q^{2 r(\mathbf{d})-h(d)} f_{\mathbf{d}} .
$$

Fix $\alpha, \beta \in \mathbb{N}^{\mathbb{Z} / n \mathbb{Z}}, \overline{\mathbf{a}} \in \mathcal{S}_{\alpha}$, and $\overline{\mathbf{b}} \in \mathcal{S}_{\beta}$. Using Lemma 13.1.b we get

$$
\gamma_{d}\left(f_{\overline{\mathbf{a}}} \circ f_{\overline{\mathbf{b}}}\right)=\sum_{\mathbf{a}, \mathbf{b}} q^{m(b, a)-m(\beta, \alpha)+2 r(\mathbf{a b})-h(d)} f_{\mathbf{a}} \circ f_{\mathbf{b}},
$$

where the sum is over all $(\mathbf{a}, \mathbf{b}) \in \mathcal{S}_{\overline{\mathbf{a}}, a} \times \mathcal{S}_{\overline{\mathbf{b}}, b}$ and all $(a, b)$ such that $\bar{a}=\alpha, \bar{b}=\beta$, and $d=a+b$. We are thus reduced to prove the following identity

(e) $m(b, a)-m(\beta, \alpha)+2 r(\mathbf{a b})-2 r(\mathbf{a})-2 r(\mathbf{b})+h(a)+h(b)-h(d)=k(b, a)$.

Set

$$
l_{+}(b, a)=\sum_{\substack{i>j \\ \bar{\imath}=\bar{\jmath}}}\left(b_{i} a_{j}+b_{j} a_{i+1}\right) \quad \text { and } \quad l_{-}(b, a)=\sum_{\substack{i<j \\ \bar{\imath}=\bar{\jmath}}}\left(b_{i} a_{j}+b_{j} a_{i+1}\right) .
$$

Then $(e)$ follows from the following equalities which are easy to prove :

$$
\begin{aligned}
& m(b, a)-m(\beta, \alpha)=-l_{+}(b, a)-l_{-}(b, a), \\
& h(a)+h(b)-h(d)=k(b, a)-l_{+}(b, a)+l_{-}(b, a), \\
& r(\mathbf{a b})-r(\mathbf{a})-r(\mathbf{b})=l_{+}(b, a) .
\end{aligned}
$$


Acknowledgements. This work was partially done while the second author was visiting the University of Chicago. The second author is grateful to the departement of Mathematics of the U.C., in particular to V. Ginzburg, for his kind invitation.

\section{References}

[A] Ariki, S.: On the decomposition numbers of the Hecke algebra of $G(m, 1, n)$. J. Math. Kyoto Univ, 36 (1996), 789-808.

[CG] Chriss, N., Ginzburg, V.: Representation theory and complex geometry. Birkhauser (1997).

[CP] Chari, V., Pressley, A.: A guide to quantum groups. Cambridge University Press (1994).

[D1] Deodhar, V.V.: On some geometric aspects of the Bruhat ordering II. The parabolic analogue of Kazhdan-Lusztig polynomials. J. Algebra, 111 (1987), 483-506.

[D2] Du, J.: A note on quantized Weyl reciprocity at roots of unity. Algebra Colloq., 2 (1995), 363-372.

[G1] Green, J.A.: Hall algebras, hereditary algebras and quantum groups. Invent. Math., 120 (1995), 361-377.

[G2] Green, R.M.: The affine $q$-Schur algebra. qalg-preprint, 9705015.

[GV] Ginzburg, V., Vasserot, E.: Langlands reciprocity for affine quantum groups of type $A_{n}$. Internat. Math. Res. Notices, 3 (1993), $67-85$.

[H] Hayashi, T.: Q-analogues of Clifford and Weyl algebras - spinor and oscillator representations of quantum enveloping algebras. Comm. Math. Phys., 127 (1990), 129-144.

[IM] Iwahori, N., Matsumoto, H.: On some Bruhat decompositions and the structure of Hecke rings of $p$-adic Chevalley groups. Pub. I.H.E.S., 25 (1965), 5-48.

[KMS] Kashiwara, M., Miwa, T., Stern, E.: Decomposition of $q$-deformed Fock space. Selecta Mathematica, New Series, 1 (1995), 787805.

[L1] Lusztig, G.: Canonical bases arising from quantized enveloping algebras. J. Amer. Math. Soc., 3 (1990), 447-498.

[L2] Lusztig, G.: Quivers, perverse sheaves and enveloping algebras. J. Amer. Math. Soc., 4 (1991), 365-421.

[L3] Lusztig, G.: Introduction to quantum groups. Birkhäuser, Progr. in Math., 110 (1993).

[L4] Lusztig, G.: Canonical bases and Hall algebras. Preprint, (1997).

[LT] Leclerc, B., Thibon, J.-Y.: Canonical bases of $q$-deformed Fock spaces. Internat. Math. Res. Notices, 9 (1996), 447-456.

[MM] Misra, K.C., Miwa, T.: Crystal base for the basic representation of $U_{q}\left(\widehat{\mathfrak{s l}}_{n}\right)$. Comm. Math. Phys., 134 (1990), 79-88. 
[N] Nakajima, I.: Instantons on ALE spaces, quiver varieties, and KacMoody algebras. Duke Math. J., 76 (1994), 365-416.

[S] Schiffmann, O.: Algèbres affines quantiques aux racines de l'unité et $K$-théorie équivariante. C. R. Acad. Sci. Paris, to appear.

[V1] Vasserot, E.: Représentations de groupes quantiques et permutations. Ann. Scient. Éc. Norm. Sup., 4ème série, 26 (1993), 747-773.

[V2] Vasserot, E.: Affine quantum groups and equivariant $K$-theory. Transformation Groups, to appear.

[VV] Varagnolo, M., Vasserot, E.: Double-loop algebras and the Fock space. Invent. Math., 133 (1998), 133-159.

Michela Varagnolo

Département de Mathématiques

Université de Cergy-Pontoise

2 Av. A. Chauvin

95302 Cergy-Pontoise Cedex

France

email: varagnol@math.pst.u-cergy.fr
Eric Vasserot

Département de Mathématiques

Université de Cergy-Pontoise

2 Av. A. Chauvin

95302 Cergy-Pontoise Cedex

France

email: vasserot@math.pst.u-cergy.fr 